\title{
Examining the relationship between free recall and immediate serial recall: Similar patterns of rehearsal and similar effects of word length, presentation rate, and articulatory suppression
}

\author{
Parveen Bhatarah \\ London Metropolitan University, London, England \\ AND \\ Geoff Ward, Jessica Smith, and Louise HaYes \\ University of Essex, Colchester, England
}

\begin{abstract}
In five experiments, rehearsal and recall phenomena were examined using the free recall and immediate serial recall (ISR) tasks. In Experiment 1, participants were presented with lists of eight words, were precued or postcued to respond using free recall or ISR, and rehearsed out loud during presentation. The patterns of rehearsal were similar in all the conditions, and there was little difference between recall in the precued and postcued conditions. In Experiment 2, both free recall and ISR were sensitive to word length and presentation rate and showed similar patterns of rehearsal. In Experiment 3, both tasks were sensitive to word length and articulatory suppression. The word length effects generalized to 6-item (Experiment 4) and 12-item (Experiment 5) lists. These findings suggest that the two tasks are underpinned by highly similar rehearsal and recall processes.
\end{abstract}

Two commonly used tests of immediate memory are the free recall task and the immediate serial recall (ISR) task. In these tasks, participants are presented with a list of words to remember, one at a time, and immediately after the end of the list, they must try to recall as many of the list items as they can. In free recall, participants are free to recall the list in any order that they like. By contrast, in ISR, participants must recall the words in exactly the same order as that in which they had been presented.

Both tasks have provided classic evidence that has been used to support the existence of a short-term memory store (STS) of limited capacity. In free recall, participants tend to recall more words from the beginning and the end of the lists (known as primacy effects and recency effects, respectively; Murdock, 1962). The primacy effect is widely assumed to reflect additional rehearsal or attention afforded to the first list items (see Rundus, 1971; Tan \& Ward, 2000), whereas the classic explanation of the recency effect is that it reflects the output of the contents of the STS (e.g., Atkinson \& Shiffrin, 1968; Glanzer, 1972). In ISR, participants' limited ability to recall a complete short sequence of verbal items in the correct order has been argued to reflect the capacity of the STS (Baddeley, Thomson, \& Buchanan, 1975; Miller, 1956).

However, there is current controversy over whether the two tasks are underpinned by common theoretical mechanisms. Currently, some authors argue that the two tasks are underpinned by common memory mechanisms (e.g., Anderson, Bothell, Lebiere, \& Matessa, 1998; Brown, Neath, \& Chater, 2007; Ward, 2001; Ward, Tan, \& Bhatarah, 2008). However, there are many accounts of free recall that have not, as yet, been applied to ISR (e.g., Davelaar, Goshen-Gottstein, Ashkenazi, Haarmann, \& Usher, 2005; Gillund \& Shiffrin, 1984; Howard \& Kahana, 2002; Raaijmakers \& Shiffrin, 1981) and many accounts of ISR that have not, as yet, been applied to free recall (e.g., Baddeley, 1986, 2000, 2007; Brown, Preece, \& Hulme, 2000; Burgess \& Hitch, 1992, 1999; Farrell \& Lewandowsky, 2002; Henson, 1998; Nairne, 1988; Page \& Norris, 1998). This divergence in the free recall and ISR literatures might seem surprising, given that both tasks were influential in establishing the concept of an STS, but it remains controversial to believe that the two tasks should be grouped together in a common theoretical framework (for a recent exchange of views on this issue, see Brown, Chater, \& Neath, 2008; Murdock, 2008).

Over the last few years, we have been examining the relationship between free recall and ISR. First, we examined whether the recency effect in free recall and ISR could be underpinned by a common STS (Bhatarah, Ward, \& Tan, 2006). In a series of experiments, we replicated and extended the work of Baddeley and Hitch (1974, 1977), who asked participants to learn a list of 16 words for free recall, with and without a concurrent six-digit memory

P. Bhatarah, p.bhatarah@londonmet.ac.uk 
load for ISR. Bhatarah et al. (2006) confirmed that the relative magnitude of the recency effect in the free recall of a 16-word list was unaffected by the presentation and recall of a different set of six digits for ISR after each and every word in the list, including the last. Bhatarah et al. (2006) found that this lack of a trade-off between recency and ISR extended to free recall under both visual and auditory presentation of the words and under overt rehearsal and fixed rehearsal instructions (Fischler, Rundus, \& Atkinson, 1970). In agreement with Baddeley and Hitch, we interpreted this finding as evidence that the recency effect in free recall and the capacity limits in ISR could not both be signatures of the same limited capacity STS. Nevertheless, subsequent findings suggested a number of similarities in the way in which the studied words were encoded and retrieved on the two tasks.

In a series of follow-up experiments (Bhatarah, Ward, $\&$ Tan, 2008), we examined whether participants used similar or different encoding strategies on the two tasks. We manipulated test expectancy, such that in precued conditions, the participants always knew the task that they would perform in advance of the list (precued free recall and precued ISR), whereas in the postcued conditions, the participants did not know the task to perform until after the last word in the list had been presented (postcued free recall and postcued ISR). We found that the shapes of the serial position curve were different for the two tasks in the postcued conditions: Postcued free recall was characterized by primacy and recency effects, whereas postcued ISR was characterized by extended primacy effects and very limited recency. Critically, there was little difference between the data from the precued conditions and the data from the postcued conditions on the two tasks. Furthermore, although the participants tended to start their recall from different serial positions in the two tasks, the degree to which there was a tendency to output in a forward order was nevertheless strikingly similar (for similar findings with slightly longer lists, see also Golomb, Peelle, Addis, Kahana, \& Wingfield, 2008). We argued that these data could be most economically explained by assuming that both tasks were underpinned by the same memory mechanisms, which were characterized by recency and forwardordered recall.

In the present series of experiments, our aim was to explore the relationship between free recall and ISR still further by examining how the two tasks were affected by factors linked directly or indirectly with rehearsal. We used the overt rehearsal technique, manipulations of presentation rate (taken from the free recall literature); and manipulations of word length and articulatory suppression (taken from the ISR literature) and applied all these techniques and manipulations to both tasks.

Critically, we compared rehearsal and recall performance on the two tasks under identical methodological conditions. We were looking for similarities and differences in the patterns of overt rehearsals and similarities and differences in the effects of the different variables on the two tasks. To the extent to which we found common patterns of data, we would be tempted to consider that the two tasks share common memory mechanisms responsible for rehearsal and recall processes.

\section{EXPERIMENT 1}

In Experiment 1, the patterns of rehearsals afforded to the list items in free recall and ISR were examined using the overt rehearsal method (Rundus \& Atkinson, 1970), in which participants are asked to say out loud any previous list items that come to mind during the study period. The overt rehearsal method has been used extensively in free recall (e.g., Brodie \& Murdock, 1977; Rundus, 1971; Tan \& Ward, 2000), using longer lists of, say, 20 words, but has also recently been examined in ISR (Tan \& Ward, 2008), using shorter lists of 6 words. In both tasks, recall is enhanced following rehearsal at slow presentation rates. However, unlike in previous studies, Experiment 1 compared performance on the two tasks directly, using equivalent lists of 8 words under identical methodological conditions.

We wished to examine whether participants might adopt different rehearsal strategies if they knew in advance the type of task with which they would be tested. Previous examinations of this manipulation (Bhatarah et al., 2008) suggested that free recall and ISR used highly similar methods of encoding, but these previous experiments did not measure the patterns of rehearsal.

Thus, each participant received four blocks of trials: (1) a block of trials in which they knew in advance that they would be tested by free recall (the precued free recall condition), (2) a block of trials in which they knew in advance that they would be tested by ISR (the precued ISR condition), and (3) two blocks of trials in which the task to be performed at test was unknown to the participant during encoding but was cued only immediately prior to recall (the blocks each contained postcued free recall and postcued ISR trials in a randomized order). On all trials, the participants saw a precue instruction, read aloud a sequence of eight visually presented words, and then saw a postcue instruction. The precue instruction either informed the participants of the task to be performed at test (the precued conditions) or else was a series of question marks (the postcued conditions); the postcue instruction always informed the participants of the task to be performed at test. An analysis of the rehearsal data across both tasks would demonstrate any similarity or difference in encoding and rehearsal between free recall and ISR.

\section{Method}

Participants. Twenty-four students from the University of Essex participated in this experiment in exchange for $£ 5$.

Materials and Apparatus. The materials for each participant consisted of 288 words randomly selected from a set of 478 words taken from the Toronto Word Pool (Friendly, Franklin, Hoffman, \& Rubin, 1982). The materials were presented, using the Supercard application, on an Apple Macintosh G4 computer with a 16-in. computer monitor. A tape recorder was used to record the participants' rehearsals and recall responses.

Design. The experiment used a within-subjects design. For each task (free recall and ISR), there were two within-subjects indepen- 
dent variables: test expectancy, with two levels (precued or postcued), and serial position of the list items, with eight levels (Serial Positions [SPs] 1, 2, 3, 4, 5, 6, 7, and 8).

Procedure. The participants were tested individually and were informed that they would be shown 4 practice lists of eight words, followed by 32 experimental lists of eight words. The experimental trials were arranged into four blocks of eight trials. In one block, the participants were always told in advance that the task would be free recall; in a different block, the participants were always told in advance that the task would be ISR; and in two other blocks, the participants did not know in advance the task to be performed. Each participant was randomly allocated to one of the 24 different orderings of the four blocks of trials. Each trial started with a warning tone, followed after $3 \mathrm{sec}$ by the precue instruction, which appeared for $3 \mathrm{sec}$ in the same position on the screen as the stimuli. The precue instruction was "Any order" or "Same order" (in the precued free recall and precued ISR conditions, respectively) or was "????????" (in the postcued conditions). After an additional $2 \mathrm{sec}$ in which the stimulus field was blank, there was a second warning tone, an additional 1-sec pause, followed by the eight words presented one at a time in the stimulus field in the center of the screen. The presentation rate was one word every $3 \mathrm{sec}$, with each word displayed for $1 \mathrm{sec}$ and an additional 2-sec interstimulus interval in which the stimulus field was blank. The participants were instructed to read aloud each word as it was presented and also to say aloud any words from the list that came to mind during the presentation of the list items. After the last item, a postcue instruction was displayed for 2 sec that read either "Any order" or "Same order" (in the free recall and ISR conditions, respectively). On the free recall trials, the participants were free to recall the words in any order that they wished, and on the ISR trials, they were instructed to recall the words in the same order as that in which they had been presented (saying "blank" when an item was forgotten, in order to maintain the serial position of subsequent recalls). The participants then heard three beeps signaling the start of the 25 -sec recall period. A tape recorder was used to record the participants' overt rehearsals, as well as to record the spoken items at recall.

\section{Results}

The results for the words recalled in free recall and ISR were analyzed separately.

Free recall. The proportions of words recalled at each serial position for the free recall tasks are shown in Figure $1 \mathrm{~A}$. A $2 \times 8$ within-subjects ANOVA with two factors-test expectancy (postcued or precued) and serial position (SP 1, 2, 3, 4, 5, 6, 7, or 8)-revealed a nonsig-

\section{A}

Experiment 1: Free Recall Precued and Postcued

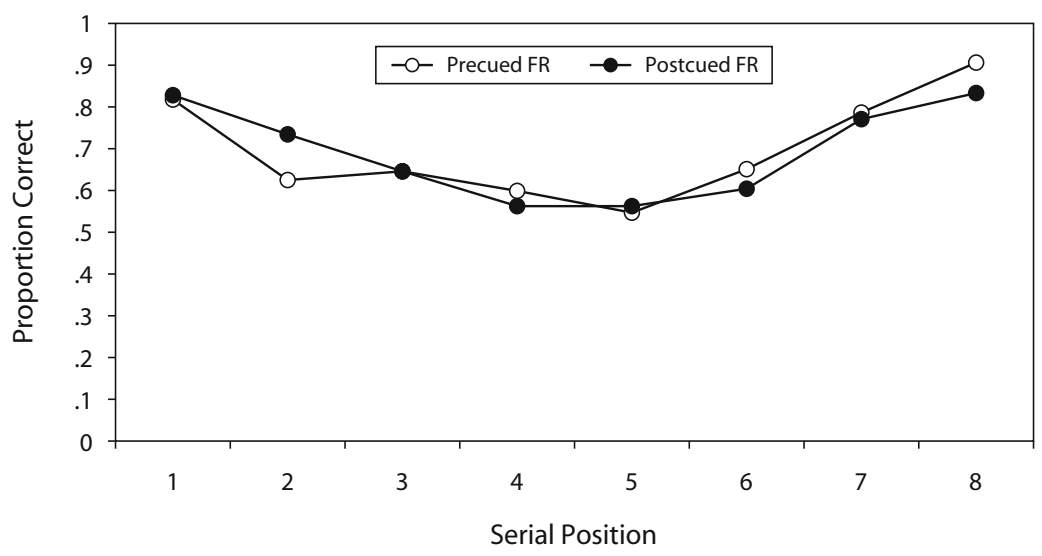

B Experiment 1: Immediate Serial Recall Precued and Postcued

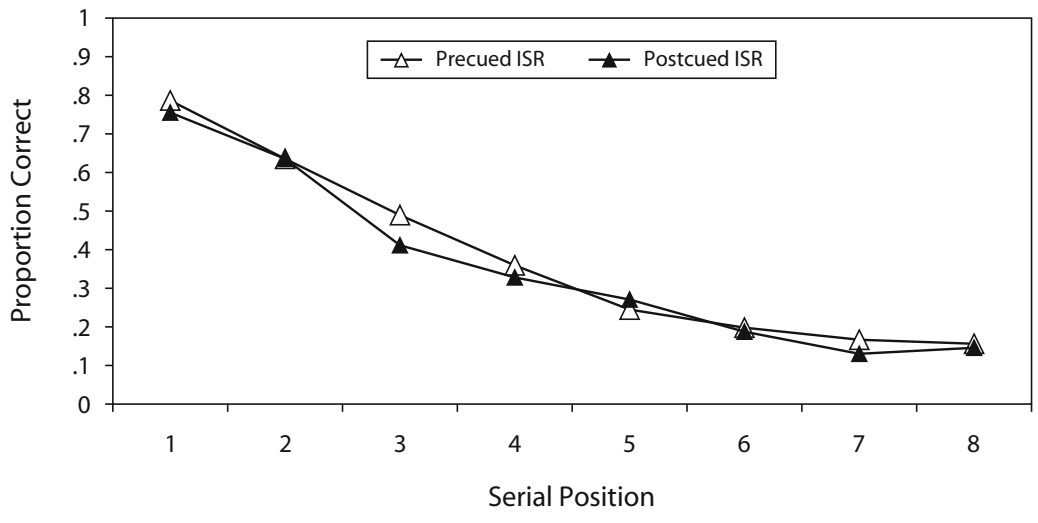

Figure 1. Experiment 1: Serial position curves for the precued and postcued free recall (FR) conditions (A) and the precued and postcued immediate serial recall (ISR) conditions (B). 
nificant main effect of test expectancy $[F(1,23)=0.95$, $\left.M S_{\mathrm{e}}=0.021, p>.05\right]$, a significant main effect of serial position $\left[F(7,161)=16.54, M S_{\mathrm{e}}=0.039, p<.01\right]$, and a nonsignificant interaction $\left[F(7,161)=1.19, M S_{\mathrm{e}}=0.031\right.$, $p>.05]$. Tukey HSD pairwise comparisons on the significant main effect of serial position revealed significant differences between SP 1 and each of SPs 2-6, between SP 2 and SP 5 (showing significant primacy), between SP 2 and SP 8, and for all pairwise comparisons between SPs 7-8 and SPs 3-6 (showing significant recency).

ISR. The proportions of words recalled in correct serial order for each serial position in the ISR tasks are displayed in Figure 1B. A $2 \times 8$ within-subjects ANOVA with two factors - test expectancy (precued and postcued) and serial position (SPs 1, 2, 3, 4, 5, 6, 7, and 8)-revealed a nonsignificant main effect of test expectancy $[F(1,23)=$ $\left.1.06, M S_{\mathrm{e}}=0.042, p>.05\right]$, a significant main effect of serial position $\left[F(7,161)=63.08, M S_{\mathrm{e}}=0.041, p<\right.$ $.001]$, and a nonsignificant interaction $[F(7,161)=0.86$, $\left.M S_{\mathrm{e}}=0.013, p>.05\right]$. Tukey HSD pairwise comparisons on the significant main effect of serial position revealed significant differences between SP 1 and SP 2, for every pairwise comparison involving SPs 1-2 and SPs 3-8, and for every pairwise comparison involving SP 4 and SPs 6-8 (showing significant primacy). There was no significant recency advantage.

Number of rehearsals. Figure 2 shows the mean number of times that an item from a given serial position was rehearsed. A $2 \times 2 \times 8$, fully within-subjects ANOVA with three factors - task (free recall or ISR), test expectancy (postcued or precued), and serial position (SP 1, 2, $3,4,5,6,7$, or 8)-revealed a nonsignificant main effect of task $\left[F(1,23)=0.348, M S_{\mathrm{e}}=0.463, p>.05\right]$, a nonsignificant main effect of test expectancy $[F(1,23)=0.248$, $\left.M S_{\mathrm{e}}=0.837, p>.05\right]$, and a significant main effect of serial position $\left[F(7,161)=54.17, M S_{\mathrm{e}}=2.489, p<.01\right]$. The task $\times$ serial position interaction $[F(7,161)=1.90$, $\left.M S_{\mathrm{e}}=0.192, p>.05\right]$ and the test expectancy $\times$ serial position interaction $\left[F(7,161)=0.186, M S_{\mathrm{e}}=0.335\right.$, $p>.05]$ did not reach significance. However, the task $\times$ test expectancy interaction was significant $[F(1,23)=$ $\left.5.00, M S_{\mathrm{e}}=0.82, p<.05\right]$, as was the task $\times$ test expectancy $\times$ serial position interaction $[F(7,161)=2.127$, $\left.M S_{\mathrm{e}}=0.579, p<.05\right]$. An examination of the simple main effects showed that when postcued, the first words in the list were rehearsed significantly more often for free recall than for ISR, and when precued, the second and third words in the list were rehearsed significantly more often for ISR than for free recall.

Patterns of rehearsal. We examined the rehearsal data further to see whether there were similar rehearsal patterns across test conditions at different times during study. We borrowed the term rehearsal set (RS) from the free recall literature (e.g., Rundus, 1971) to denote that set of rehearsals that are voiced in the interstimulus interval that follows the presentation of each word. Using this nomenclature, RS 1 refers to the set of words rehearsed after the presentation of the first word in the list, RS 2 refers to the set of words rehearsed after the presentation of the second word in the list, and so on, up to RS 8, which refers to the set of words rehearsed after the presentation of the eighth (and in this study, final) word in the list prior to the recall cue. Figure 3 shows which words from the list were rehearsed at least once in each RS in each of the experimental conditions. In each panel, the $y$-axis represents the serial position of the words that were rehearsed, and there are separate curves for the rehearsals afforded in the intervals (RSs) following the eight different words in the list. Panels A, B, C, and D demonstrate the proportions of words rehearsed in the precued free recall, postcued free recall, precued ISR, and postcued ISR conditions, respectively. For all four conditions, there were similar declines in the proportion of words rehearsed at later RSs and similar distributions of words rehearsed across different RSs for the four conditions. The participants nearly always obeyed the instruction to read out loud the currently presented list item. However, it is not strictly the case that these utterances should be treated as a true rehearsal. Therefore, in Figure 3, the currently presented word was counted as a genuine rehearsal only if it was said out loud at least twice at some point in the rehearsal set (the first utterance of the stimulus was deemed to be simply obeying task instruc-

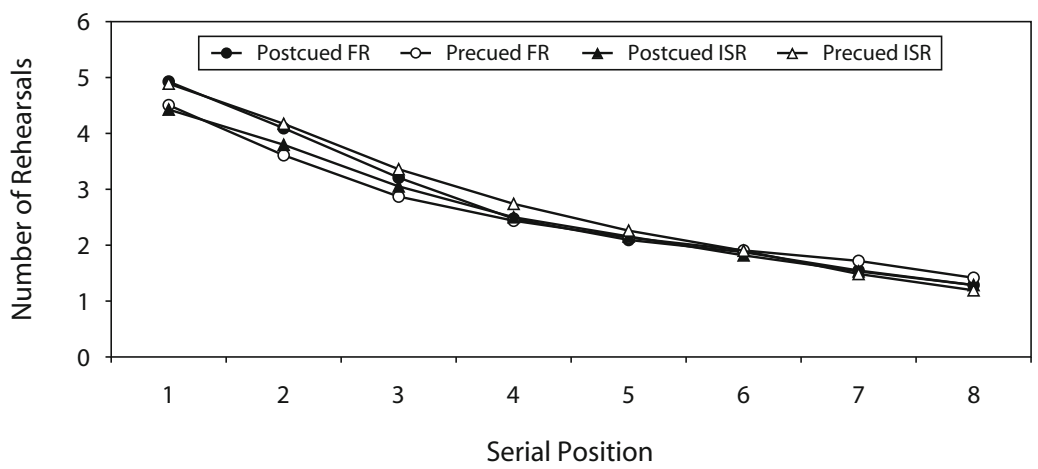

Figure 2. Experiment 1: Mean number of rehearsals afforded to the words in the lists tested in the precued and postcued free recall (FR) conditions and the precued and postcued immediate serial recall (ISR) conditions. 


\section{A Proportion of Words Rehearsed}

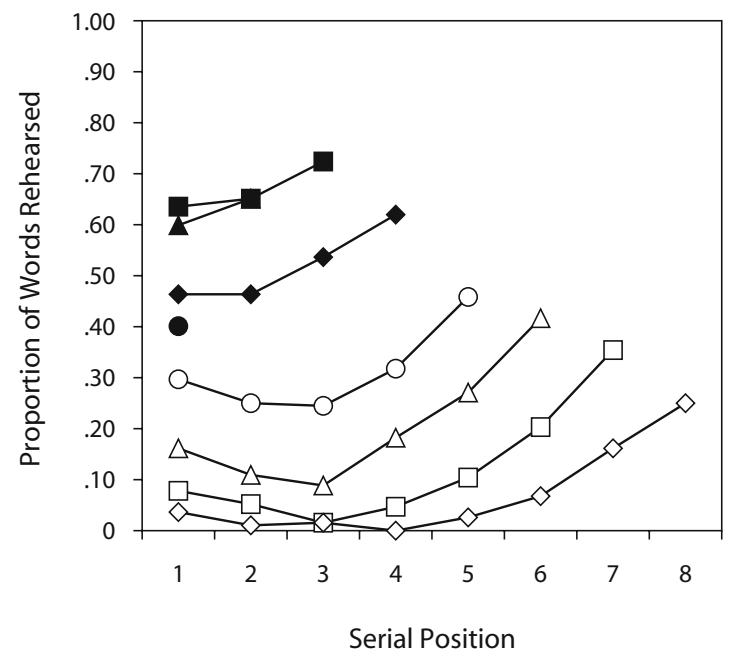

Precued ISR

\section{Proportion of Words Rehearsed}

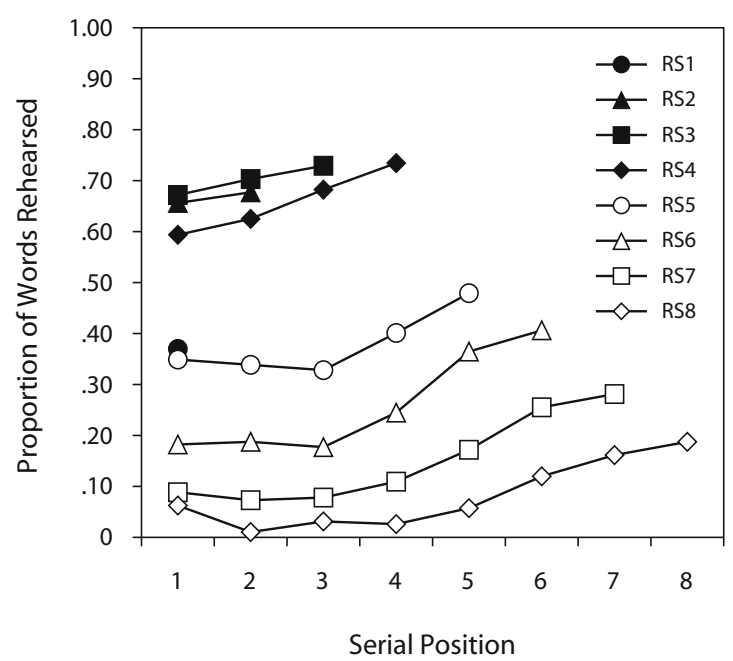

Postcued Free Recall

B Proportion of Words Rehearsed

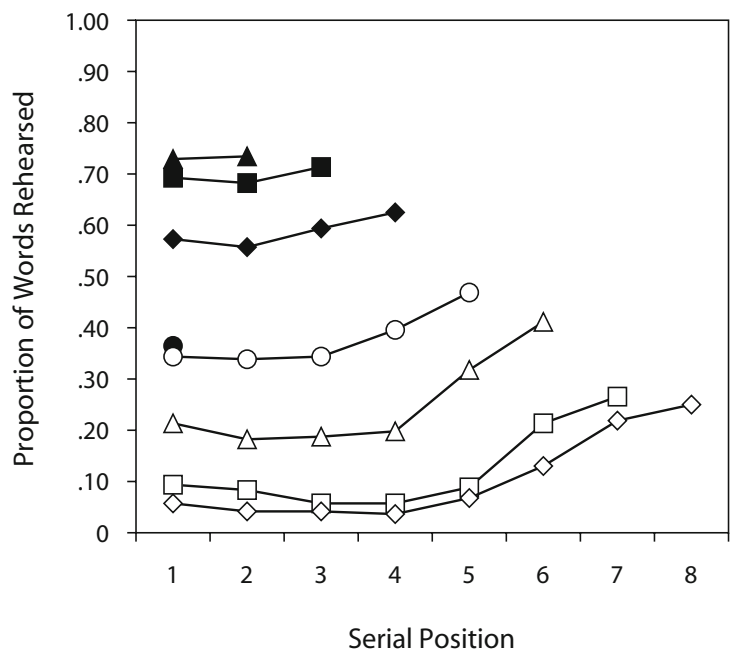

Postcued ISR

D Proportion of Words Rehearsed

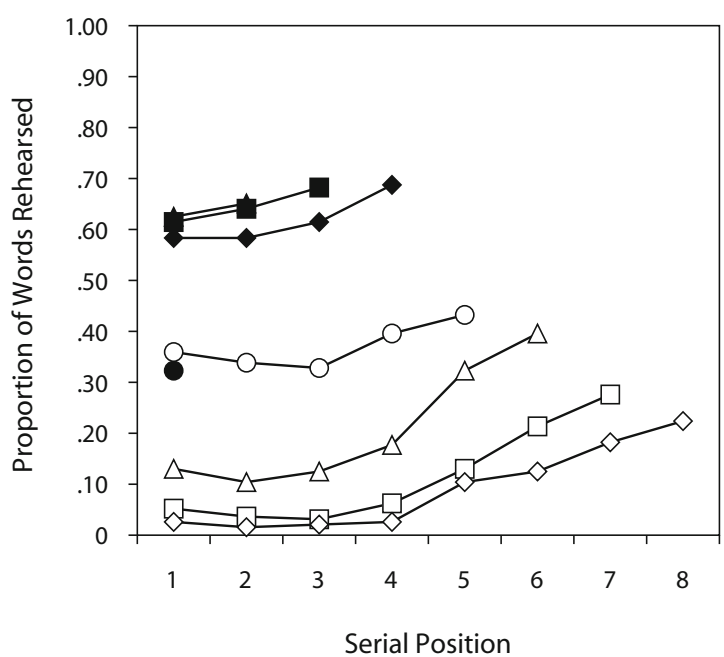

Figure 3. Experiment 1: Mean proportions of trials on which words from different serial positions were rehearsed at least once. (A and B) Patterns of rehearsals in the precued and postcued free recall conditions, respectively. (C and D) Patterns of rehearsals in the precued and postcued immediate serial recall (ISR) conditions, respectively. The eight separate lines in each panel plot the rehearsals in the eight different rehearsal sets (RSs); a rehearsal set is simply the set of words that are rehearsed after each of the eight words in the list, so that RS 6 refers to the set of words rehearsed after the sixth word in the list.

tions, but the second and further utterances of the stimulus in the RS were deemed to be a genuine rehearsal).

Analysis of output order. We examined the order in which the words were output at recall in the four conditions. Table 1 shows the count of all the words recalled in the four conditions, tabulated by the output order and the serial position of the words in the list. Consistent with task instructions, the participants who were required to perform ISR initiated their recall with the first word in the list. They also tended to proceed forward in their recall, leading to an increased density of responses along the leading diagonals. Note that recall performance decreased with output position: Later list items were neither recalled in their correct position nor recalled in their incorrect position, suggesting that the primacy effects in ISR reflect a loss in the accessibility of later list items. By contrast, the dominant tendency for those who were required to perform free recall was to initiate output with a word from the 
Table 1

Analysis of Output Order in Experiment 1: Number of Words Recalled As a Function of Task, Expectancy Condition, and Serial Position

\begin{tabular}{|c|c|c|c|c|c|c|c|c|c|}
\hline \multirow{2}{*}{$\begin{array}{c}\text { Serial } \\
\text { Position }\end{array}$} & \multicolumn{8}{|c|}{ Output Position } & \multirow[b]{2}{*}{ Tota } \\
\hline & 1 & 2 & 3 & 4 & 5 & 6 & 7 & 8 & \\
\hline \multicolumn{10}{|c|}{ Precued Immediate Serial Recall } \\
\hline SP 1 & 151 & 3 & 1 & 2 & 1 & 1 & & & 15 \\
\hline SP 2 & 3 & 122 & 5 & 2 & & 1 & & 1 & 134 \\
\hline SP 3 & 6 & 15 & 94 & 4 & 3 & 3 & & 1 & 126 \\
\hline SP 4 & 7 & 10 & 19 & 69 & 9 & 2 & 1 & 1 & 118 \\
\hline SP 5 & 8 & 7 & 13 & 23 & 47 & 4 & 1 & 1 & 104 \\
\hline SP 6 & 4 & 9 & 9 & 21 & 28 & 38 & 5 & 1 & 115 \\
\hline SP 7 & 2 & 8 & 14 & 7 & 17 & 17 & 32 & 2 & 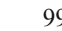 \\
\hline SP 8 & 1 & 2 & 9 & 16 & 10 & 18 & 16 & 30 & 102 \\
\hline Total & 182 & 176 & 164 & 144 & 115 & 84 & 55 & 37 & 957 \\
\hline \multicolumn{10}{|c|}{ Postcued Immediate Serial Recall } \\
\hline SP 1 & 145 & 2 & 3 & 3 & 3 & & & & 156 \\
\hline SP 2 & 8 & 122 & 6 & 3 & 1 & 1 & 1 & & 142 \\
\hline SP 3 & 8 & 13 & 79 & 12 & 5 & & & & 117 \\
\hline SP 4 & 5 & 8 & 15 & 63 & 6 & 3 & & & 100 \\
\hline SP 5 & 6 & 10 & 20 & 20 & 52 & 4 & 2 & 1 & 115 \\
\hline SP 6 & 2 & 5 & 13 & 12 & 21 & 36 & 2 & 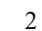 & 93 \\
\hline SP 7 & 3 & 10 & 8 & 13 & 18 & 21 & 25 & 4 & 102 \\
\hline SP 8 & 3 & 4 & 17 & 13 & 18 & 19 & 15 & 28 & 117 \\
\hline Total & 180 & 174 & 161 & 139 & 124 & 84 & 45 & 35 & 942 \\
\hline \multicolumn{10}{|c|}{ Precued Free Recall } \\
\hline SP 1 & 45 & 12 & 19 & 25 & 34 & 11 & 9 & 2 & 157 \\
\hline SP 2 & 2 & 24 & 13 & 27 & 22 & 19 & 9 & 4 & 120 \\
\hline SP 3 & 3 & 7 & 40 & 21 & 2 & 14 & 6 & 4 & 12 \\
\hline SP 4 & 2 & 12 & 16 & 27 & 28 & 16 & 11 & 3 & 115 \\
\hline SP 5 & 18 & 10 & 17 & 29 & 9 & 13 & 6 & 3 & 105 \\
\hline SP 6 & 22 & 27 & 27 & 18 & 16 & 11 & 2 & 2 & 125 \\
\hline SP 7 & 36 & 54 & 26 & 17 & 8 & 7 & 2 & 1 & 151 \\
\hline SP 8 & 64 & 43 & 30 & 16 & 8 & 8 & 4 & 1 & 174 \\
\hline Total & 192 & 189 & 188 & 180 & 154 & 99 & 49 & 20 & 1,071 \\
\hline \multicolumn{10}{|c|}{ Postcued Free Recall } \\
\hline SP 1 & 56 & 10 & 27 & 28 & 0 & 9 & 5 & 1 & 159 \\
\hline SP 2 & 5 & 48 & 1 & 3 & & 7 & 5 & 2 & 141 \\
\hline SP 3 & 5 & 8 & 47 & 20 & 18 & 17 & 8 & 1 & 124 \\
\hline SP 4 & 5 & 10 & 9 & 39 & 14 & 17 & 10 & 4 & 108 \\
\hline SP 5 & 19 & 11 & 12 & 12 & 23 & 19 & 8 & 4 & 108 \\
\hline SP 6 & 25 & 25 & 22 & 8 & 15 & 13 & 6 & 2 & 11 \\
\hline SP 7 & 31 & 44 & 20 & 20 & 18 & 5 & 5 & 5 & 1 \\
\hline SP 8 & 45 & 34 & 36 & 16 & 14 & 8 & 4 & 3 & 160 \\
\hline Total & 191 & 190 & 188 & 178 & 149 & 95 & 51 & 22 & 1,06 \\
\hline
\end{tabular}

second half of the list, although there was also a significant minority of trials in both the precued and postcued free recall conditions in which the participants started to recall from SP 1.

\section{Discussion}

There were two main findings. First, there were different serial position curves for free recall and ISR in the postcued blocks of trials, which followed trends that were very similar to those for the precued trials of each task. Free recall performance across the serial position curve demonstrated primacy and recency effects, and ISR performance was characterized by extended primacy effects and no recency. These data suggest that the differences in the shapes of the serial position curves were due more to the retrieval characteristics of the different tasks than to differences in strategic encoding.
Second, the rehearsal schedules obtained from participant rehearsal protocols indicated similar, although not identical, patterns of rehearsal across both tasks. In all four conditions, there was a strong trend for early items to be rehearsed the most and for the numbers of rehearsals of study items to decrease as a function of serial position. Furthermore, the distributions of rehearsals were very similar for the four conditions across the different serial positions within the lists at the different rehearsal sets.

Nevertheless, there were small but significant increases in the number of rehearsals afforded to the second and third words in the precued ISR condition, relative to the precued free recall condition, which may reflect a small strategic difference in the patterns of rehearsal in ISR, where there is a greater necessity to maintain the accessibility of early items at the time of test. We can, however, propose no logical reason why the number of rehearsals might be expected to change between the two postcued conditions, suggesting that these small differences, at least, must be attributed to Type I error.

Therefore, although there were only minor differences in the patterns of rehearsals on the two tasks, there remained large differences in the patterns of recall. This combination of findings prompts important questions regarding the function of rehearsal: What does it do, and how does rehearsal affect recall? Our supposition is that the function of rehearsal in these immediate memory tasks is to keep as many of the words in the list as accessible as possible at the time of test. As Tan and Ward (2008) have demonstrated, when participants perform ISR at medium or slower presentation rates, their ability to access the start of the list decreases if they do not rehearse but increases if they do rehearse. Similarly, Tan and Ward (2000) have also shown that early list items in free recall that are not rehearsed show a dramatically reduced probability of being recalled, but the recall probability of early list items increases greatly following increases in the number, recency, and distribution of rehearsals.

However, accessibility at the time of test is not the only factor determining the shapes of the serial position curves. The postcued data from Experiment 1 clearly demonstrate that participants can exert some control over the order in which they recall. The analysis of the output order in Experiment 1 shows that participants who are postcued to perform ISR can elect to initiate their recall with the very first list item in ISR (on the majority of trials) and can continue in a forward serial order, leading to patterns of primacy effects. By contrast, participants who are postcued to perform free recall can elect to output the later list items first, thereby raising the level of recency (for additional demonstrations of the flexibility of participants to recall in different orders, see Bhatarah et al., 2008; Tan \& Ward, 2007). The postcued free recall data show that there was enhanced accessibility of the recency items at the time of test, and the postcued ISR data show that this accessibility greatly decreased following the output of earlier list items. This suggests that the later list items in ISR are particularly susceptible to output interference (see also Tan \& Ward, 2007), a major factor determining the different shapes of the serial position curves for the two tasks. 


\section{EXPERIMENT 2}

In Experiment 2, we again examined the patterns of rehearsals and recalls in free recall and ISR and, additionally, manipulated variables commonly linked to the ability to rehearse - namely, presentation rate (taken from free recall literature) and word length (taken from the ISR literature).

There is considerable evidence that free recall performance increases with slower presentation rates (e.g., Brodie \& Murdock, 1977; Glanzer \& Cunitz, 1966; Tan \& Ward, 2000). It is widely assumed that a slower presentation rate gives participants more opportunity to rehearse. However, there are different interpretations as to the ways in which rehearsals may influence recall and also different views about the mechanisms underpinning rehearsal. According to the classic dual-store account of free recall (e.g., Atkinson \& Shiffrin, 1968, 1971; Glanzer, 1972), rehearsal is performed within the short-term buffer within the STS. At the slower rate, participants rehearse the words more often, and the increased numbers of rehearsals cause the words to be more strongly associated in the long-term memory store (LTS), resulting in superior recall. Subsequent authors have additionally emphasized the importance of recency (Brodie \& Murdock, 1977; Tan \& Ward, 2000), and the distribution of rehearsals (Modigliani \& Hedges, 1987; Tan \& Ward, 2000) on later recall. Laming (2006) has argued that an STS account of rehearsal may not be necessary but, rather, that the mechanisms that underpin rehearsal during encoding are the same as those used at retrieval during recall.

The effect of presentation rate on ISR is more varied. In some cases, a slower presentation rate gives rise to poorer ISR performance (e.g., Conrad \& Hille, 1958; Posner, 1964), whereas in other cases, a slower rate gives rise to superior ISR performance (e.g., Mackworth, 1962; Tan \& Ward, 2008). The recent analyses of Tan and Ward (2008) suggested that participants often attempt cumulative, forward-ordered rehearsal at slower rates but have time only to repeat aloud the currently presented item at faster rates. Although slower rates led to superior recall overall (presumably because of the additional benefits of forwardordered cumulative rehearsal), post hoc analyses showed that when one considers only that subset of trials on which there was no forward-ordered rehearsal, slower rates led to worse recall than did faster rates. The data can readily be accounted for by phonological loop accounts of ISR (e.g., Baddeley, 1986; Burgess \& Hitch, 1999; Page \& Norris, 1998): When participants maintained the list items through rehearsal at the slower rates, this led to improved recall, whereas if these items were not rehearsed, the slower rates led to greater retention intervals for the earlier items, and so these items were subject to greater trace decay.

To consider now the effects of word length, there is considerable evidence and widespread agreement that ISR performance decreases when the number of syllables in the words in the list are increased from one up to three, or even up to as many as five (Baddeley, 1986; Baddeley et al., 1975). The word length effect in ISR has attracted considerable theoretical interest because it is claimed that it provides evidence in support of the concept of trace decay (Baddeley, 2007; Cowan, 2005; Mueller, Seymour, Kieras, \& Meyer, 2003; Schweikert \& Boruff, 1986), although many alternative explanations have since been proposed (e.g., Brown \& Hulme, 1995; Hulme et al., 2006; Hulme, Suprenant, Bireta, Stuart, \& Neath, 2004; Lewandowsky \& Farrell, 2000; Lewandowsky \& Oberauer, 2008; Nairne, 2002; Neath \& Brown, 2006; Neath \& Nairne, 1995; Tehan \& Tolan, 2007).

The working memory model (Baddeley, 1986) assumes that the word length effect is a key empirical finding supporting the existence of the phonological loop mechanism of working memory that is used for both rehearsal and recall in ISR. According to the phonological loop model, the word length effect arises because longer words are rehearsed more slowly during encoding, so that the phonological memory traces of long words are subject to more decay, and may also arise at output, because longer words will take longer to recall than shorter words. A number of authors have emphasized the importance of output delay in the word length effect in ISR (e.g., Dosher \& $\mathrm{Ma}, 1998)$, and this is consistent with the findings that the word length effect is reduced when the output delay is reduced in probed recall (Avons, Wright, \& Pammer, 1994) and still present with presentation rates so rapid that rehearsal during presentation is unlikely (Coltheart \& Langdon, 1998). However, a number of authors have questioned whether articulatory duration is the critical factor (Service, 1998; Tolan \& Tehan, 2005) or whether the effect of articulatory duration might be restricted to a limited stimulus set (Bireta, Neath, \& Surprenant, 2006; Caplan, Rochon, \& Waters, 1992; Lovatt, Avons, \& Masterson, 2000, 2002).

By contrast, there has been far less interest in whether a word length effect exists in free recall. Early investigations scrutinized whether the recency effect in free recall was particularly sensitive to word length, in order to see whether the recency effect and ISR might share a common STS explanation, but no such heightened sensitivity was found (e.g., Watkins, 1972). Nevertheless, those studies that have examined the effect of word length on free recall have also shown a detrimental overall effect of increased word length when monosyllabic words were compared with polysyllabic words (Russo \& Grammatopoulou, 2003; Salthouse, 1980; Watkins, 1972).

Although some published studies have examined the effects of presentation rate and word length on free recall and ISR, direct comparisons are complicated by the fact that the methods of presentation used in free recall and ISR tasks have rarely, if ever, been identical in terms of list length, presentation rate, and the stimulus sets that were used. Experiment 2 sought to circumvent these problems by examining the effects of presentation rate and word length on free recall and ISR under identical methods of presentation. Because one of the main aims of the study was to examine the role of rehearsal in these tasks, we continued with the overt rehearsal method that we used in Experiment 1 (Rundus \& Atkinson, 1970) throughout all the conditions. To our knowledge, this is the first attempt to explicitly measure rehearsal while manipulating word length in an immediate memory task. 
In Experiment 2, we used three different word lengths and two different presentation rates. The participants were presented with lists of eight words for free recall and ISR. Each word was presented visually on the computer screen for 1 or $2.5 \mathrm{sec}$. The participants read each word aloud as it was presented and, at test, recalled the list items in any order that they liked (free recall) or in the same order as that in which they had been presented (ISR). The lists of words selected for the experiment consisted of words of one, three, or five syllables from a large open set of words.

\section{Method}

\section{Participants}

Twenty-four students from the University of Essex participated in this experiment in exchange for course credit. None had taken part in Experiment 1.

\section{Materials and Apparatus}

The materials consisted of a pool of 205 one-syllable words, a pool of 205 three-syllable words, and a pool of 205 five-syllable words. The words were selected from the Oxford Psycholinguistic database (Quinlan, 1992), with frequencies of occurrence of 2-88 per million on the basis of Kučera and Francis's (1967) norms, and the mean frequencies for each word length were matched. The materials were presented, using the Supercard application, on an Apple Macintosh G3 computer, with a 16-in. monitor.

\section{Design}

The experiment used a within-subjects design with three independent variables: presentation rate with two levels (fast or slow), word length with three levels (long, medium, or short words), and serial position with eight levels (SPs 1-8).

\section{Procedure}

The participants were tested individually and were informed that they would be shown 2 practice lists of eight words, followed by 72 experimental lists of eight words for free recall and ISR. The experimental lists were divided into four blocks of 18 trials. In order to keep the task requirements as easy to follow for the participants as possible, the same task (free recall or ISR) was always used in the first two blocks, and the same presentation rate (fast or slow) was always used in the first and third blocks. There were, therefore, four permissible block orders, and these four orders were counterbalanced across participants. The participants were instructed as to which task to perform prior to the presentation of the stimuli in each block. For the 18 trials in each block, there were 6 trials of eight long words, 6 trials of eight medium words, and 6 trials of eight long words. The order of the 18 trials was randomized within each block. On all the trials, the participants were instructed to read aloud each word as it was presented on the screen and also to rehearse out loud any words that came to mind during the study phase. Each trial began with a warning tone, followed after $1 \mathrm{sec}$ by a series of eight words. The words were presented individually in the middle of the computer screen for $1 \mathrm{sec}$ in the fast presentation condition and $2.5 \mathrm{sec}$ in the slow presentation condition. After the last word from the study list had been presented, a series of beeps signaled the beginning of the $25-\mathrm{sec}$ recall period for verbal recall. A tape recorder was used to record the participants' rehearsal protocols and recall performance.

\section{Results}

\section{Free Recall}

Serial position. Figures $4 \mathrm{~A}$ and $4 \mathrm{~B}$ show the serial position curves for free recall in the fast and slow con- ditions, respectively. A $2 \times 3 \times 8$ fully within-subjects ANOVA with three factors - presentation rate (fast or slow), word length (short, medium, or long words), and serial position (SPs 1-8) - revealed a significant main effect of presentation rate $\left[F(1,23)=34.83, M S_{\mathrm{e}}=\right.$ $0.032, p<.001]$, a significant main effect of word length $\left[F(2,46)=125.4, M S_{\mathrm{e}}=0.035, p<.001\right]$, and a significant main effect of serial position $[F(7,161)=91.85$, $\left.M S_{\mathrm{e}}=0.08, p<.001\right]$.

The presentation rate $\times$ word length interaction was not significant $\left[F(2,46)=0.622, M S_{\mathrm{e}}=0.025, p>.05\right]$. However, the presentation rate $\times$ serial position interaction was significant $\left[F(7,161)=6.30, M S_{\mathrm{e}}=0.038, p<\right.$ $.001]$, as was the word length $\times$ serial position interaction $\left[F(1,23)=10.39, M S_{\mathrm{e}}=0.025, p<.01\right]$. Finally, the three-way presentation rate $\times$ word length $\times$ serial position interaction was not found to be significant $\left[F(14,322)=1.27, M S_{\mathrm{e}}=0.03, p>.05\right]$.

A simple main effects analysis performed on the interaction between presentation rate and serial position revealed that the recall advantage for the words presented at a slow rate was limited to the first four serial positions. A simple main effects analysis performed on the interaction between word length and serial position showed that the significant word length effects were limited to the first six serial positions.

Number of rehearsals. Figures $5 \mathrm{~A}$ and $5 \mathrm{~B}$ show the numbers of rehearsals afforded to words in each of the serial positions for the fast and slow conditions, respectively, in the free recall task. A $2 \times 3 \times 8$ fully withinsubjects ANOVA with three factors - presentation rate (fast or slow), word length (long, medium, or short), and serial position (1-8) - revealed a significant main effect of presentation rate $\left[F(1,23)=29.70, M S_{\mathrm{e}}=2.235, p<\right.$ $.001]$, a significant main effect of word length $[F(2,46)=$ $\left.31.03, M S_{\mathrm{e}}=0.470, p<.001\right]$, and a significant main effect of serial position $\left[F(7,161)=19.73, M S_{\mathrm{e}}=0.520\right.$, $p<.001]$. All two-way interactions were significant: presentation rate $\times$ word length interaction $[F(2,46)=$ $\left.27.25, M S_{\mathrm{e}}=0.307, p<.001\right]$, presentation rate $\times$ serial position $\left[F(7,161)=29.26, M S_{\mathrm{e}}=0.214, p<.001\right]$, and word length $\times$ serial position $\left[F(14,322)=20.89, M S_{\mathrm{e}}=\right.$ $0.075, p<.001]$. The three-way interaction was also significant $\left[F(14,322)=19.42, M S_{\mathrm{e}}=0.056, p<.001\right]$.

A simple main effects analysis of the three-way interaction revealed that at a fast presentation rate, there was little rehearsal beyond that instructed when the words are read aloud upon presentation. At the fast rate, there was no significant increase in rehearsals for early list items and no significant increases in rehearsals for shorter words. By contrast, there were far more overt rehearsals at the slow presentation rate. At the slow rate, short words were rehearsed more often, overall, than were medium words, which, in turn, were rehearsed more often than were long words. Within the short and medium word lengths, there were significantly more rehearsals for early list items than for later list items. There was, however, little rehearsal of the long words presented at any serial position at the slow presentation rate. 


\section{Experiment 2: Free Recall Fast Presentation Rate}

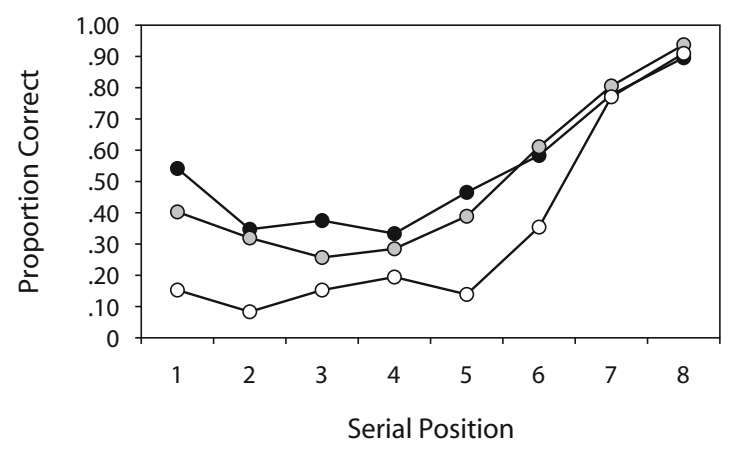

C Experiment 2: Immediate Serial

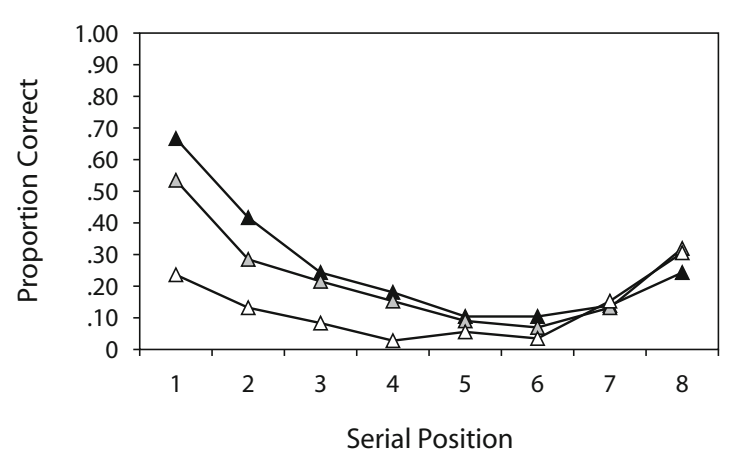

\section{B Slow Presentation Rate}

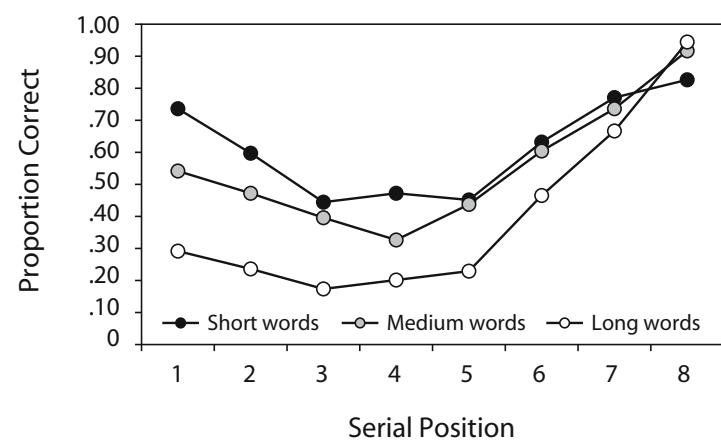

Experiment 2: Immediate Serial Recall Slow Presentation Rate

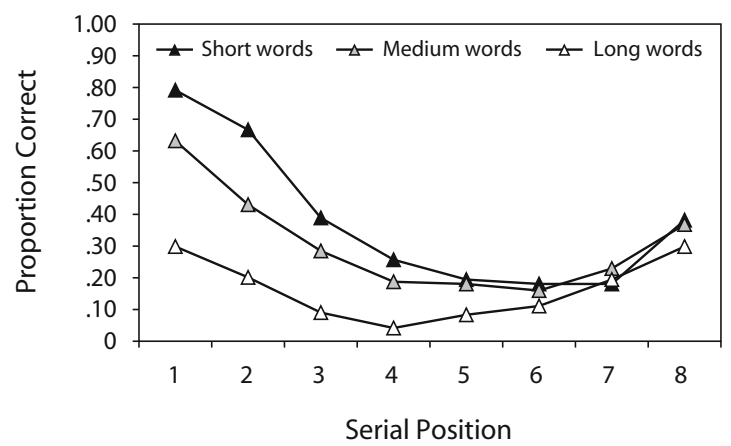

Figure 4. Experiment 2: Serial position curves for the lists of short, medium, and long words presented at a fast rate (A) or a slow rate (B) for free recall. The lower panels show the serial position curves for the lists of short, medium, and long words presented at a fast rate (C) and a slow rate (D) for immediate serial recall.

\section{Immediate Serial Recall}

Serial position. Figures $4 \mathrm{C}$ and $4 \mathrm{D}$ show the serial position curves for the ISR task at the fast and slow presentation rates, respectively. A $2 \times 3 \times 8$ fully withinsubjects ANOVA with three factors - presentation rate (fast or slow), word length (long, medium, or short), and serial position (1-8) - revealed a significant main effect of presentation rate $\left[F(1,23)=21.51, M S_{\mathrm{e}}=0.085, p<\right.$ $.001]$, a significant main effect of word length $[F(2,46)=$ 61.54, $\left.M S_{\mathrm{e}}=0.050, p<.001\right]$, and a significant main effect of serial position $\left[F(7,161)=25.88, M S_{\mathrm{e}}=0.118\right.$, $p<.001]$. The presentation rate $\times$ word length interaction was found to be significant $\left[F(2,46)=5.52, M S_{\mathrm{e}}=\right.$ $0.029, p<.01]$, as was the word length $\times$ serial position interaction $\left[F(14,322)=13.73, M S_{\mathrm{e}}=0.027, p<.001\right]$. The presentation rate $\times$ serial position interaction did not reach significance $\left[F(7,161)=1.45, M S_{\mathrm{e}}=0.029, p>\right.$ $.05]$. The three-way presentation rate $\times$ word length $\times$ serial position interaction also did not reach significance $\left[F(14,322)=0.834, M S_{\mathrm{e}}=0.019, p>.05\right]$.

A simple main effects analysis of the presentation rate $\times$ word length interaction showed an advantage for the slow presentation rate over the fast presentation rate when the word length was short or medium, but not when it was fast. In addition, the extent of the word length effect was exaggerated at slow rates, relative to fast rates. A simple main effects analysis of the word length $\times$ serial position interaction revealed a simple main effect of word length only at the first four serial positions and significant primacy and recency effects at all three word lengths, with the degree of primacy enhanced at shorter word lengths.

Number of rehearsals. Figures $5 \mathrm{C}$ and $5 \mathrm{D}$ show the numbers of rehearsals afforded to words in each of the serial positions for the fast and slow conditions, respectively, in ISR. A $2 \times 3 \times 8$ fully within-subjects ANOVA with three factors - presentation rate (fast or slow), word length (long, medium, or short), and serial position (1-8) - revealed a significant main effect of presentation rate $\left[F(1,23)=53.37, M S_{\mathrm{e}}=1.477, p<.001\right]$, a significant main effect of word length $\left[F(2,46)=37.22, M S_{\mathrm{e}}=\right.$ $0.455, p<.001]$, and a significant main effect of serial position $\left[F(7,161)=19.75, M S_{\mathrm{e}}=1.228, p<.001\right]$. All two-way interactions were found to be significant: presentation rate $\times$ word length $\left[F(2,46)=30.62, M S_{\mathrm{e}}=0.276\right.$, $p<.001]$, presentation rate $\times$ serial position $[F(7,161)=$ $\left.45.34, M S_{\mathrm{e}}=0.182, p<.001\right]$, and word length $\times$ serial 



\section{Experiment 2: Immediate Serial}

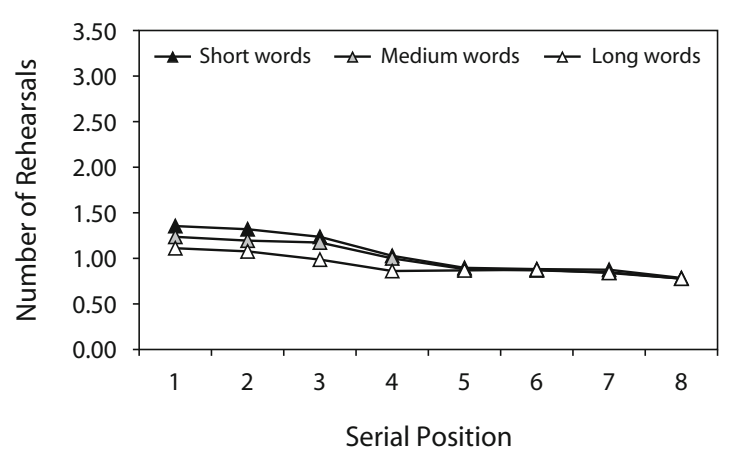

Dexperiment 2: Immediate Serial Recall Slow Presentation Rate

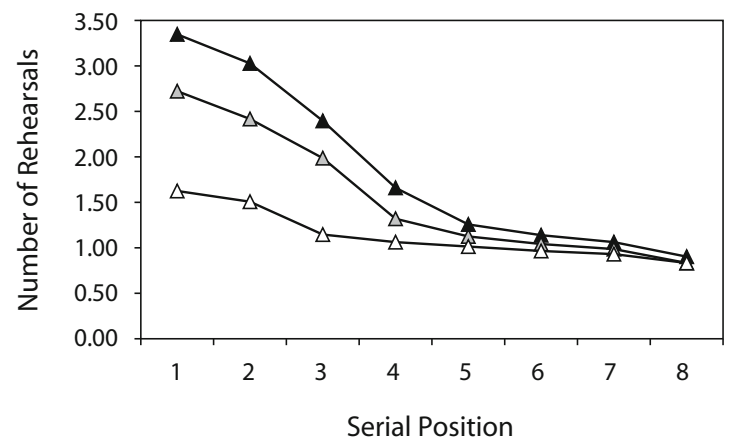

Figure 5. Experiment 2: Mean number of rehearsals afforded to the words in the lists tested in the fast (A) and slow (B) free recall conditions and the fast $(C)$ and slow $(D)$ immediate serial recall conditions.

position $\left[F(14,322)=22.03, M S_{\mathrm{e}}=0.088, p<.001\right]$. The three-way interaction was also found to be significant $\left[F(14,322)=17.42, M S_{\mathrm{e}}=0.060, p<.001\right]$.

A simple main effects analysis of the three-way interaction showed that at the fast presentation rate, there was little or no additional overt rehearsal at any word length or serial position. By contrast, at the slow presentation rate, there were significant increases in the number of overt rehearsals, but these were limited to the short- and medium-length words, which occupied early serial positions in the list.

\section{Analysis of Output Order}

Tables 2A-2D show the count of the words recalled in all the different conditions, tabulated by the output order and the serial position of the words in the list. The dominant tendency for the participants who were required to perform ISR was to initiate their recall with the first word in the list and then proceed forward in their recall (note the increased density of responses along the leading diagonals). However, the ability to access the first item in the list was affected by both the presentation rate and the word length: The participants were much more likely to initiate recall with the first word in the list with shorter words and at the slower presentation rate. The dominant tendency for the participants who were required to perform free recall was to initiate their recall from the second half of the list. However, on a minority of the trials, there was also a tendency for the first word in the list to be recalled first in the free recall conditions. The size of this minority tendency increased at the slower rate and with shorter words.

\section{Discussion}

There were four main findings in Experiment 2. First, the patterns of rehearsal afforded to the words during the free recall task were strikingly similar to those afforded to words during the ISR task. In both tasks, there was little or no extra rehearsal of words presented at the fast rate. However, at the slow presentation rate, there was considerable rehearsal in both tasks for short and medium-length words that occupied early list positions.

Second, despite the similar patterns of rehearsals, the shapes of the serial position curves differed for the two tasks. In the free recall task, there was greater recency and less primacy, whereas in the ISR task, there was greater primacy and less recency. The demonstration that the two 
Free Recall and Immediate Serial Recall

Table 2A

Analysis of Output Order in Experiment 2: Number of Words Recalled at Each Output Position As a Function of Experimental Task, Word Length, and Serial Position in the Immediate Serial Recall Fast Presentation Condition

\begin{tabular}{|c|c|c|c|c|c|c|c|c|c|}
\hline \multirow{2}{*}{$\begin{array}{c}\text { Serial } \\
\text { Position }\end{array}$} & \multicolumn{8}{|c|}{ Output Position } & \multirow[b]{2}{*}{ Total } \\
\hline & 1 & 2 & 3 & 4 & 5 & 6 & 7 & 8 & \\
\hline \multicolumn{10}{|c|}{ Long Words } \\
\hline SP 1 & 34 & 2 & 3 & 0 & 1 & 0 & 0 & 1 & 41 \\
\hline SP 2 & 0 & 19 & 3 & 2 & 0 & 0 & 0 & 0 & 24 \\
\hline SP 3 & 1 & 3 & 12 & 3 & 0 & 4 & 1 & 0 & 24 \\
\hline $\mathrm{SP} 4$ & 1 & 3 & 2 & 4 & 2 & 0 & 1 & 0 & 13 \\
\hline SP 5 & 3 & 3 & 4 & 4 & 8 & 3 & 1 & 1 & 27 \\
\hline SP 6 & 5 & 8 & 5 & 3 & 9 & 5 & 4 & 0 & 39 \\
\hline SP 7 & 1 & 5 & 6 & 8 & 1 & 7 & 22 & 0 & 50 \\
\hline SP 8 & 10 & 1 & 0 & 2 & 10 & 7 & 8 & 44 & 88 \\
\hline Total & 55 & 44 & 35 & 26 & 31 & 26 & 37 & 46 & 306 \\
\hline \multicolumn{10}{|c|}{ Medium Words } \\
\hline SP 1 & 77 & 3 & 0 & 2 & 0 & 0 & 0 & 0 & 82 \\
\hline SP 2 & 1 & 41 & 4 & 2 & 1 & 1 & 0 & 1 & 51 \\
\hline SP 3 & 3 & 6 & 31 & 5 & 2 & 0 & 1 & 0 & 48 \\
\hline SP 4 & 3 & 5 & 10 & 22 & 8 & 0 & 1 & 1 & 50 \\
\hline SP 5 & 3 & 2 & 11 & 11 & 13 & 3 & 1 & 1 & 45 \\
\hline SP 6 & 1 & 10 & 5 & 7 & 8 & 10 & 4 & 2 & 47 \\
\hline SP 7 & 3 & 6 & 10 & 3 & 10 & 14 & 19 & 3 & 68 \\
\hline SP 8 & 6 & 2 & 1 & 6 & 7 & 6 & 7 & 46 & 83 \\
\hline Total & 97 & 75 & 72 & 58 & 49 & 34 & 33 & 54 & 474 \\
\hline \multicolumn{10}{|c|}{ Short Words } \\
\hline SP 1 & 96 & 2 & 3 & 0 & 0 & 0 & 0 & 0 & 101 \\
\hline SP 2 & 5 & 60 & 6 & 3 & 1 & 1 & 0 & 0 & 76 \\
\hline SP 3 & 2 & 10 & 35 & 6 & 5 & 0 & 0 & 0 & 58 \\
\hline SP 4 & 1 & 5 & 11 & 26 & 0 & 2 & 0 & 1 & 46 \\
\hline SP 5 & 4 & 3 & 12 & 11 & 15 & 3 & 4 & 1 & 53 \\
\hline SP 6 & 2 & 6 & 7 & 12 & 10 & 15 & 3 & 0 & 55 \\
\hline SP 7 & 3 & 6 & 7 & 4 & 11 & 8 & 20 & 2 & 61 \\
\hline SP 8 & 6 & 4 & 2 & 5 & 8 & 9 & 4 & 35 & 73 \\
\hline Total & 119 & 96 & 83 & 67 & 50 & 38 & 31 & 39 & 523 \\
\hline
\end{tabular}

tasks give rise to rather different serial position curves despite similar encoding strategies supports the findings obtained in Experiment 1.

Third, there were clear effects of word length for both tasks and presentation rates, and the word length effects were limited to the early list positions in all four conditions. It should be noted that at the fast presentation rate, there was little or no rehearsal of the words, yet there were still clear word length effects, consistent with the idea that some aspects of word length effects may arise at recall, even when rehearsal is reduced or prevented during the encoding of the list (Baddeley \& Lewis, 1984; Coltheart \& Langdon, 1998).

Finally, it was interesting to note that there were similarities and subtle differences in the interactions between presentation rate, word length, and serial position across the tasks. For both tasks, the effect of word length was far greater at early serial positions. Specifically, the difference between the proportion of words recalled for the short words and for the long words at SP 1 was +.417 for free recall and +.462 for ISR, but these advantages gradually declined throughout the list, so that the advantage was reversed at SP 8 in free recall $(-.066)$ and was reduced close to zero in ISR $(+.010)$.
Table 2B

Analysis of Output Order in Experiment 2: Number of Words Recalled at Each Output Position As a Function of Experimental Task, Word Length, and Serial Position in the Immediate Serial Recall Slow Presentation Condition

\begin{tabular}{|c|c|c|c|c|c|c|c|c|c|}
\hline \multirow{2}{*}{$\begin{array}{c}\text { Serial } \\
\text { Position } \\
\end{array}$} & \multicolumn{8}{|c|}{ Output Position } & \multirow[b]{2}{*}{ Total } \\
\hline & 1 & 2 & 3 & 4 & 5 & 6 & 7 & 8 & \\
\hline \multicolumn{10}{|c|}{ Long Words } \\
\hline SP 1 & 43 & 1 & 1 & 0 & 2 & 1 & 0 & 0 & 48 \\
\hline SP 2 & 7 & 29 & 2 & 3 & 1 & 0 & 2 & 0 & 44 \\
\hline SP 3 & 1 & 4 & 13 & 3 & 0 & 0 & 1 & 0 & 22 \\
\hline SP 4 & 0 & 1 & 6 & 6 & 3 & 0 & 0 & 1 & 17 \\
\hline SP 5 & 1 & 1 & 1 & 10 & 12 & 2 & 3 & 0 & 30 \\
\hline SP 6 & 1 & 1 & 4 & 2 & 5 & 16 & 2 & 2 & 33 \\
\hline SP 7 & 0 & 2 & 1 & 0 & 7 & 8 & 28 & 3 & 49 \\
\hline SP 8 & 3 & 2 & 2 & 1 & 3 & 6 & 8 & 43 & 75 \\
\hline Total & 56 & 41 & 30 & 25 & 33 & 33 & 44 & 49 & 318 \\
\hline \multicolumn{10}{|c|}{ Medium Words } \\
\hline SP 1 & 91 & 2 & 1 & 0 & 2 & 1 & 0 & 0 & 97 \\
\hline SP 2 & 0 & 62 & 3 & 1 & 1 & 2 & 0 & 0 & 69 \\
\hline SP 3 & 2 & 7 & 41 & 3 & 0 & 1 & 1 & 0 & 55 \\
\hline SP 4 & 5 & 2 & 10 & 27 & 5 & 1 & 0 & 1 & 51 \\
\hline SP 5 & 1 & 7 & 4 & 7 & 26 & 2 & 3 & 1 & 51 \\
\hline SP 6 & 1 & 1 & 2 & 8 & 12 & 23 & 3 & 1 & 51 \\
\hline SP 7 & 0 & 0 & 2 & 5 & 7 & 11 & 33 & 4 & 62 \\
\hline SP 8 & 1 & 0 & 2 & 4 & 5 & 3 & 8 & 53 & 76 \\
\hline Total & 101 & 81 & 65 & 55 & 58 & 44 & 48 & 60 & 512 \\
\hline \multicolumn{10}{|c|}{ Short Words } \\
\hline SP 1 & 114 & 3 & 3 & 0 & 0 & 0 & 0 & 0 & 120 \\
\hline SP 2 & 0 & 96 & 4 & 0 & 0 & 0 & 0 & 0 & 100 \\
\hline SP 3 & 1 & 7 & 56 & 7 & 1 & 0 & 2 & 0 & 74 \\
\hline SP 4 & 1 & 3 & 13 & 37 & 2 & 0 & 0 & 1 & 57 \\
\hline SP 5 & 1 & 1 & 7 & 18 & 28 & 3 & 0 & 1 & 59 \\
\hline SP 6 & 0 & 0 & 3 & 8 & 11 & 26 & 4 & 2 & 54 \\
\hline SP 7 & 1 & 0 & 0 & 2 & 9 & 8 & 26 & 0 & 46 \\
\hline SP 8 & 0 & 1 & 2 & 1 & 6 & 9 & 6 & 55 & 80 \\
\hline Total & 118 & 111 & 88 & 73 & 57 & 46 & 38 & 59 & 590 \\
\hline
\end{tabular}

However, the effect of presentation rate interacted with different variables on the two tasks, perhaps reflecting the different output orders that were typically used on the two tasks. In free recall, participants are free to output in any order that they choose, and they typically output the recency items first. The recency items are, therefore, almost always correctly recalled, regardless of the presentation rate, and it is only the early list items that benefit from a slow rate (perhaps because they were rehearsed toward the end of the list, increasing their accessibility; Tan \& Ward, 2000). In ISR, participants must initiate recall with the first list item and then output in a forward order. In an eight-item list, recall of the first word in the list is difficult in the absence of rehearsal, and the last words in the list cannot benefit from immediate recall but must wait until earlier items have been output. Therefore, all serial positions benefited equally from the slow rate, but the short words benefited more than the long words from the slow rate (because the short words were particularly easy to rehearse at the slow rates).

Overall, there were a number of similarities between the patterns of overt rehearsals and the effects of word length and presentation rate on performance on the free recall and ISR tasks. This line of enquiry was continued in Experiment 3. 
Table 2C

Analysis of Output Order in Experiment 2: Number of Words Recalled at Each Output Position As a Function of Experimental Task, Word Length, and Serial Position in the Free Recall Fast Presentation Condition

\begin{tabular}{|c|c|c|c|c|c|c|c|c|c|}
\hline \multirow{2}{*}{$\begin{array}{c}\text { Serial } \\
\text { Position }\end{array}$} & \multicolumn{8}{|c|}{ Output Position } & \multirow[b]{2}{*}{ Total } \\
\hline & 1 & 2 & 3 & 4 & 5 & 6 & 7 & 8 & \\
\hline \multicolumn{10}{|c|}{ Long Words } \\
\hline SP 1 & 7 & 4 & 7 & 4 & 0 & 0 & 0 & 0 & 22 \\
\hline SP 2 & 2 & 0 & 6 & 2 & 2 & 0 & 0 & 0 & 12 \\
\hline SP 3 & 0 & 6 & 10 & 6 & 0 & 0 & 0 & 0 & 22 \\
\hline SP 4 & 4 & 3 & 12 & 8 & 1 & 0 & 0 & 0 & 28 \\
\hline SP 5 & 2 & 3 & 11 & 1 & 2 & 1 & 0 & 0 & 20 \\
\hline SP 6 & 10 & 16 & 20 & 5 & 0 & 0 & 0 & 0 & 51 \\
\hline SP 7 & 22 & 73 & 15 & 1 & 0 & 0 & 0 & 0 & 111 \\
\hline SP 8 & 95 & 27 & 8 & 1 & 0 & 0 & 0 & 0 & 131 \\
\hline Total & 142 & 132 & 89 & 28 & 5 & 1 & 0 & 0 & 397 \\
\hline \multicolumn{10}{|c|}{ Medium Words } \\
\hline SP 1 & 17 & 5 & 9 & 15 & 7 & 4 & 1 & 0 & 58 \\
\hline SP 2 & 0 & 6 & 14 & 12 & 10 & 3 & 1 & 0 & 46 \\
\hline SP 3 & 2 & 5 & 9 & 12 & 6 & 3 & 0 & 0 & 37 \\
\hline SP 4 & 1 & 6 & 10 & 14 & 8 & 2 & 0 & 0 & 41 \\
\hline SP 5 & 4 & 4 & 26 & 19 & 2 & 1 & 0 & 0 & 56 \\
\hline SP 6 & 18 & 13 & 36 & 17 & 3 & 1 & 0 & 0 & 88 \\
\hline SP 7 & 25 & 74 & 9 & 7 & 1 & 0 & 0 & 0 & 116 \\
\hline SP 8 & 76 & 27 & 24 & 5 & 3 & 0 & 0 & 0 & 135 \\
\hline Total & 143 & 140 & 137 & 101 & 40 & 14 & 2 & 0 & 577 \\
\hline \multicolumn{10}{|c|}{ Short Words } \\
\hline SP 1 & 34 & 5 & 11 & 9 & 11 & 7 & 1 & 0 & 78 \\
\hline SP 2 & 3 & 16 & 4 & 11 & 10 & 3 & 3 & 0 & 50 \\
\hline SP 3 & 4 & 4 & 16 & 14 & 8 & 7 & 0 & 1 & 54 \\
\hline SP 4 & 2 & 7 & 12 & 16 & 8 & 1 & 2 & 0 & 48 \\
\hline SP 5 & 9 & 9 & 21 & 19 & 3 & 5 & 1 & 0 & 67 \\
\hline SP 6 & 18 & 13 & 26 & 19 & 7 & 1 & 0 & 0 & 84 \\
\hline SP 7 & 23 & 57 & 20 & 6 & 6 & 0 & 0 & 0 & 112 \\
\hline SP 8 & 51 & 27 & 21 & 15 & 7 & 8 & 0 & 0 & 129 \\
\hline Total & 144 & 138 & 131 & 109 & 60 & 32 & 7 & 1 & 622 \\
\hline
\end{tabular}

\section{EXPERIMENT 3}

In Experiment 3, the effects of word length and articulatory suppression on free recall and ISR were examined. It is well known that the word length effect is normally found in ISR when the words are presented with both visual and auditory presentation and that ISR performance is also affected by articulatory suppression (Levy, 1971; Murray, 1968). However, when participants are required to recall lists of short and long words with and without articulatory suppression, the word length effect persists with auditory presentation but is eliminated (Baddeley, Lewis, \& Vallar, 1984; Baddeley et al., 1975; Longoni, Richardson, \& Aiello, 1993) or reduced (La Pointe \& Engle, 1990; Russo \& Grammatopoulou, 2003) under visual presentation.

There is, by comparison, very little research in which the effects of articulatory suppression on free recall have been examined. In an early study by Richardson and Baddeley (1975), a common STS explanation of the recency effect in free recall and ISR was examined. The researchers examined whether the recency effect in free recall might be particularly susceptible to articulatory suppression. However, they found that the recency effect was not differentially
Table 2D

Analysis of Output Order in Experiment 2: Number of Words Recalled at Each Output Position As a Function of Experimental Task, Word Length, and Serial Position in the Free Recall Slow Presentation Condition

\begin{tabular}{crrrrrrrrr}
\hline Serial & \multicolumn{7}{c}{ Output Position } & \\
\cline { 2 - 6 } Position & 1 & 2 & 3 & 4 & 5 & 6 & 7 & 8 & Total \\
\hline \multicolumn{8}{c}{ Long Words } \\
SP 1 & 4 & 4 & 17 & 12 & 5 & 0 & 0 & 0 & 42 \\
SP 2 & 1 & 3 & 16 & 8 & 5 & 1 & 0 & 0 & 34 \\
SP 3 & 0 & 5 & 9 & 8 & 2 & 1 & 0 & 0 & 25 \\
SP 4 & 3 & 5 & 9 & 11 & 0 & 1 & 0 & 0 & 29 \\
SP 5 & 5 & 9 & 6 & 6 & 6 & 0 & 1 & 0 & 33 \\
SP 6 & 9 & 20 & 31 & 4 & 3 & 0 & 0 & 0 & 67 \\
SP 7 & 18 & 64 & 12 & 2 & 0 & 0 & 0 & 0 & 96 \\
SP 8 & 103 & 29 & 3 & 0 & 1 & 0 & 0 & 0 & 136 \\
Total & 143 & 139 & 103 & 51 & 22 & 3 & 1 & 0 & 462
\end{tabular}

\begin{tabular}{|c|c|c|c|c|c|c|c|c|c|}
\hline \multicolumn{10}{|c|}{ Medium Words } \\
\hline SP 1 & 16 & 7 & 16 & 24 & 8 & 3 & 4 & 0 & 78 \\
\hline SP 2 & 7 & 15 & 11 & 17 & 12 & 6 & 0 & 0 & 68 \\
\hline SP 3 & 2 & 6 & 19 & 12 & 11 & 7 & 0 & 0 & 57 \\
\hline SP 4 & 4 & 4 & 12 & 14 & 8 & 5 & 0 & 0 & 47 \\
\hline SP 5 & 10 & 7 & 15 & 16 & 12 & 3 & 0 & 0 & 63 \\
\hline SP 6 & 7 & 24 & 35 & 13 & 6 & 2 & 0 & 0 & 87 \\
\hline SP 7 & 20 & 52 & 22 & 6 & 3 & 3 & 0 & 0 & 106 \\
\hline SP 8 & 78 & 25 & 10 & 11 & 7 & 1 & 0 & 0 & 132 \\
\hline Total & 144 & 140 & 140 & 113 & 67 & 30 & 4 & 0 & 638 \\
\hline \multicolumn{10}{|c|}{ Short Words } \\
\hline SP 1 & 50 & 10 & 14 & 12 & 12 & 8 & 0 & 0 & 106 \\
\hline SP 2 & 4 & 38 & 11 & 14 & 8 & 7 & 4 & 0 & 86 \\
\hline SP 3 & 1 & 8 & 27 & 8 & 14 & 4 & 2 & 0 & 6 \\
\hline SP 4 & 5 & 1 & 14 & 26 & 16 & 5 & 1 & 0 & \\
\hline SP 5 & 8 & 6 & 9 & 19 & 14 & 5 & 3 & 1 & 65 \\
\hline SP 6 & 10 & 24 & 23 & 11 & 11 & 10 & 2 & 0 &  \\
\hline SP 7 & 20 & 32 & 29 & 12 & 8 & 5 & 4 & 1 & 111 \\
\hline SP 8 & 45 & 22 & 12 & 20 & 10 & 7 & 2 & 1 & \\
\hline Total & 143 & 141 & 139 & 122 & 93 & 51 & 18 & 3 & \\
\hline
\end{tabular}

sensitive to articulatory suppression but, rather, that free recall was impaired at all serial positions with lists of 10 and 16 words. A later study by Russo and Grammatopoulou (2003) also showed effects of articulatory suppression on immediate and delayed free recall of 14-word lists but did not report the effects at different serial positions.

Therefore, there seems to be some existing evidence that one might expect to see effects of word length and articulatory suppression on recall in both tasks, However, in Experiment 3, we sought to examine the effects of these variables on the two tasks, using identical methods of presentation.

\section{Method}

Participants. Twenty-four students from the University of Essex participated in this experiment in exchange for course credit or $£ 5$. None had taken part in the earlier experiments.

Materials and Apparatus. The materials for each participant consisted of 416 words: 4 practice lists and 48 experimental lists of 8 words each. The 48 experimental lists were divided into two categories: short (one-syllable) and long (five-syllable) words. The stimuli were selected at random for each participant from the onesyllable and five-syllable sets of words described in Experiment 2. The materials were presented, using the Supercard application, on an Apple Macintosh G3 computer, with a 16-in. monitor. 
Design. This experiment used a within-subjects design with three independent variables: articulation, with two levels (articulatory suppression or no suppression), word length, with two levels (short or long words), and serial position, with eight levels (SPs 1-8).

Procedure. The participants were tested individually and were informed that they would be shown 4 practice lists of eight words, followed by 48 experimental lists of eight words. The experimental lists were divided into four blocks of 12 trials. In order to keep the task requirements as easy to follow for the participants as possible, the same task (free recall or ISR) was always used in the first two blocks, and the same articulation condition (quiet or suppression) was always used in the first and third blocks. There were, therefore, four permissible block orders, and these four orders were counterbalanced across participants. The participants were instructed as to which task to perform prior to the presentation of the stimuli of each block. In the suppression trials, the participants were instructed to repeat aloud the word "the," whereas in the nonsuppression trials, they were instructed to "keep quiet" during the presentation of the list. Within the 12 trials of each block, there were 6 trials of eight long words and 6 trials of eight short words. The order of the trials within each block was randomized. On all the trials, there was a warning tone, followed after $3 \mathrm{sec}$ by a series of eight words. The words were presented individually in the middle of the computer screen for $2 \mathrm{sec}$. A series of beeps signaled the beginning of the recall period, $1 \mathrm{sec}$ after the last word from the study list was presented on the computer screen. All the participants were given $25 \mathrm{sec}$ to recall out loud as many words from the list as they could, in any order in the free recall task and in serial order in the ISR task. A tape recorder was used to record the participants' vocalizations during the interstimulus intervals (thus ensuring that suppression instructions were adhered to) and during recall performance.

\section{Results}

Free recall. The proportions of items recalled at each serial position for each word length and articulation condition in free recall are shown in Figure 6A. A $2 \times 2 \times 8$ fully within-subjects ANOVA with three factors - articulation condition (articulatory suppression or quiet), word length (short or long), and serial position (1-8) - revealed a significant main effect of articulation condition $\left[F(1,23)=19.45, M S_{\mathrm{e}}=0.026, p<.001\right]$, a significant main effect of word length $[F(1,23)=123.31$, $\left.M S_{\mathrm{e}}=0.058, p<.001\right]$, and a significant main effect of serial position $\left[F(7,161)=48.20, M S_{\mathrm{e}}=0.064, p<\right.$ $.001]$. The articulation condition $\times$ word length interaction was found to be significant $\left[F(1,23)=10.45, M S_{\mathrm{e}}=\right.$ $0.014, p<.01]$, as was the word length $\times$ serial position interaction $\left[F(7,161)=6.68, M S_{\mathrm{e}}=0.043, p<.001\right]$. The articulation condition $\times$ serial position interaction was not found to be significant $\left[F(7,161)=2.05, M S_{\mathrm{e}}=\right.$ $0.036, p>.05]$. The three-way articulation condition $\times$ word length $\times$ serial position interaction was significant $\left[F(7,161)=4.61, M S_{\mathrm{e}}=0.03, p<.001\right]$.

A simple main effects analysis of the three-way interaction showed an advantage for the quiet condition over articulatory suppression for short words at SPs $1-5$ but no significant simple main effect of articulatory suppression for later serial positions or for long words. Word length effects were also evident at all the serial positions with articulatory suppression, but only with early and middle serial positions in the quiet condition. There were significant recency effects in all the conditions and significant primacy with the short words, especially under quiet conditions.

\section{Experiment 3: Free Recall. Effects of \\ A Word Length and Articulatory Suppression}

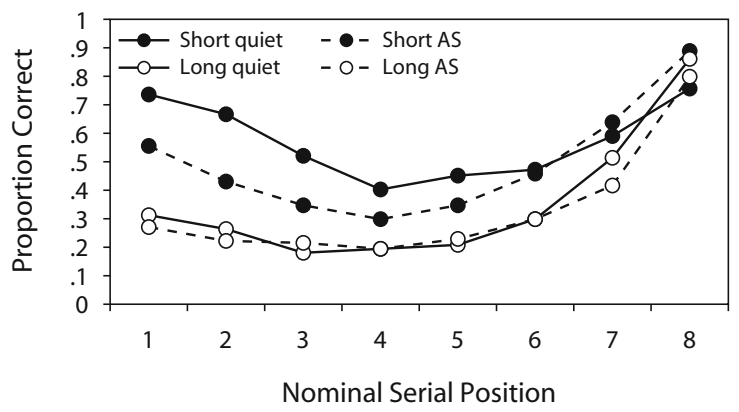

B

Experiment 3: ISR. Effects of Word Length and Articulatory Suppression



Figure 6. Experiment 3: (A) Serial position curves for the lists of short and long words presented for free recall under quiet and articulatory suppression (AS) conditions. (B) Serial position curves under quiet and AS conditions for the lists of short and long words presented for immediate serial recall (ISR).

ISR. The proportions of items recalled in their correct serial position for each word length and articulation condition in ISR are illustrated in Figure 6B. A $2 \times 2 \times 8$ fully within-subjects ANOVA with three factors-articulation (articulatory suppression or quiet), word length (short or long), and serial position (1-8) - revealed a significant main effect of articulation $[F(1,23)=24.87$, $\left.M S_{\mathrm{e}}=0.058, p<.001\right]$, a significant main effect of word length $\left[F(1,23)=92.77, M S_{\mathrm{e}}=0.050, p<.001\right]$, and a significant main effect of serial position $[F(7,161)=$ 88.14, $\left.M S_{\mathrm{e}}=0.037, p<.001\right]$. All two-way interactions were significant: articulation $\times$ word length $[F(1,23)=$ $\left.8.61, M S_{\mathrm{e}}=0.028, p<.01\right]$, articulation $\times$ serial position $\left[F(7,161)=6.31, M S_{\mathrm{e}}=0.022, p<.001\right]$, and word length $\times$ serial position $\left[F(7,161)=12.03, M S_{\mathrm{e}}=0.029\right.$, $p<.001]$. The three-way activity $\times$ word length $\times$ serial position interaction was also found to be significant $\left[F(7,161)=4.96, M S_{\mathrm{e}}=0.024, p<.001\right]$.

A simple main effects analysis of the three-way interaction indicated that word length was found to have an effect on early and middle words in both articulation conditions, that articulatory suppression reduced recall for 
Table 3A

Analysis of Output Order in Experiment 3: Number of Words Recalled at Each Output Position As a Function of Experimental Task, Word Length, and Serial Position in the Immediate Serial Recall Articulatory Suppression Condition

\begin{tabular}{|c|c|c|c|c|c|c|c|c|c|}
\hline \multirow{2}{*}{$\begin{array}{c}\text { Serial } \\
\text { Position }\end{array}$} & \multicolumn{8}{|c|}{ Output Position } & \multirow[b]{2}{*}{ Total } \\
\hline & 1 & 2 & 3 & 4 & 5 & 6 & 7 & 8 & \\
\hline \multicolumn{10}{|c|}{ Long Words } \\
\hline SP 1 & 59 & 1 & 2 & 1 & 1 & 1 & 0 & 0 & 65 \\
\hline SP 2 & 0 & 39 & 4 & 1 & 1 & 1 & 1 & 0 & 47 \\
\hline SP 3 & 1 & 3 & 10 & 2 & 0 & 0 & 0 & 0 & 16 \\
\hline SP 4 & 0 & 0 & 6 & 11 & 2 & 0 & 1 & 0 & 20 \\
\hline SP 5 & 0 & 0 & 0 & 4 & 7 & 0 & 1 & 0 & 12 \\
\hline SP 6 & 1 & 0 & 0 & 4 & 6 & 11 & 2 & 2 & 26 \\
\hline SP 7 & 0 & 3 & 1 & 1 & 4 & 8 & 9 & 0 & 26 \\
\hline SP 8 & 4 & 1 & 0 & 1 & 3 & 3 & 5 & 21 & 38 \\
\hline Total & 65 & 47 & 23 & 25 & 24 & 24 & 19 & 23 & 250 \\
\hline \multicolumn{10}{|c|}{ Short Words } \\
\hline SP 1 & 93 & 3 & 0 & 1 & 1 & 0 & 0 & 0 & 98 \\
\hline SP 2 & 1 & 67 & 4 & 0 & 1 & 0 & 0 & 0 & 73 \\
\hline SP 3 & 1 & 5 & 41 & 2 & 0 & 1 & 1 & 1 & 52 \\
\hline SP 4 & 2 & 2 & 0 & 20 & 3 & 2 & 0 & 1 & 30 \\
\hline SP 5 & 0 & 1 & 2 & 5 & 7 & 3 & 0 & 0 & 18 \\
\hline SP 6 & 1 & 1 & 0 & 2 & 6 & 13 & 3 & 0 & 26 \\
\hline SP 7 & 1 & 2 & 1 & 1 & 1 & 6 & 22 & 3 & 37 \\
\hline SP 8 & 3 & 1 & 1 & 0 & 2 & 6 & 3 & 42 & 58 \\
\hline Total & 102 & 82 & 49 & 31 & 21 & 31 & 29 & 47 & 392 \\
\hline
\end{tabular}

Table 3C

Analysis of Output Order in Experiment 3: Number of Words Recalled at Each Output Position As a Function of Experimental Task, Word Length, and Serial Position in the Free Recall Articulatory Suppression Condition

\begin{tabular}{|c|c|c|c|c|c|c|c|c|c|}
\hline \multirow{2}{*}{$\begin{array}{c}\text { Serial } \\
\text { Position }\end{array}$} & \multicolumn{8}{|c|}{ Output Position } & \multirow[b]{2}{*}{ Total } \\
\hline & 1 & 2 & 3 & 4 & 5 & 6 & 7 & $\overline{8}$ & \\
\hline \multicolumn{10}{|c|}{ Long Words } \\
\hline SP 1 & 5 & 14 & 14 & 6 & 0 & 0 & 0 & 0 & 39 \\
\hline SP 2 & 5 & 9 & 11 & 5 & 2 & 0 & 0 & 0 & 32 \\
\hline SP 3 & 0 & 9 & 11 & 7 & 3 & 1 & 0 & 0 & 31 \\
\hline SP 4 & 5 & 8 & 9 & 4 & 2 & 0 & 0 & 0 & 28 \\
\hline SP 5 & 4 & 7 & 15 & 5 & 1 & 1 & 0 & 0 & 33 \\
\hline SP 6 & 11 & 18 & 10 & 3 & 1 & 0 & 0 & 0 & 43 \\
\hline SP 7 & 13 & 36 & 10 & 1 & 0 & 0 & 0 & 0 & 60 \\
\hline SP 8 & 98 & 13 & 4 & 0 & 0 & 0 & 0 & 0 & 115 \\
\hline Total & 141 & 114 & 84 & 31 & 9 & 2 & 0 & 0 & 381 \\
\hline \multicolumn{10}{|c|}{ Short Words } \\
\hline SP 1 & 16 & 12 & 18 & 19 & 8 & 6 & 1 & 0 & 80 \\
\hline SP 2 & 3 & 15 & 19 & 12 & 8 & 5 & 0 & 0 & 62 \\
\hline SP 3 & 3 & 8 & 15 & 11 & 10 & 1 & 2 & 0 & 50 \\
\hline SP 4 & 5 & 3 & 8 & 12 & 8 & 5 & 2 & 0 & 43 \\
\hline SP 5 & 9 & 12 & 7 & 10 & 6 & 4 & 2 & 0 & 50 \\
\hline SP 6 & 8 & 23 & 16 & 11 & 6 & 1 & 0 & 1 & 66 \\
\hline SP 7 & 19 & 43 & 18 & 8 & 4 & 0 & 0 & 0 & 92 \\
\hline SP 8 & 81 & 20 & 12 & 11 & 2 & 2 & 0 & 0 & 128 \\
\hline Total & 144 & 136 & 113 & 94 & 52 & 24 & 7 & 1 & 571 \\
\hline
\end{tabular}

short but not long words for all but the later list items, and that there were significant primacy effects in all four conditions. However, primacy was more marked with short words, particularly for the lists of short words in the quiet condition.

Analysis of output order. Tables $3 \mathrm{~A}-3 \mathrm{D}$ show the count of the words recalled in all the different conditions
Table 3B

Analysis of Output Order in Experiment 3: Number of Words Recalled at Each Output Position As a Function of Experimental Task, Word Length, and Serial Position in the Immediate Serial Recall Quiet Condition

\begin{tabular}{lrrrrrrrrr}
\hline Serial & \multicolumn{7}{c}{ Output Position } \\
\cline { 2 - 6 } Position & 1 & 2 & 3 & 4 & 5 & 6 & 7 & 8 & Total \\
\hline & \multicolumn{7}{c}{ Long Words } \\
SP 1 & 71 & 1 & 1 & 0 & 1 & 0 & 2 & 0 & 76 \\
SP 2 & 2 & 47 & 2 & 0 & 1 & 0 & 0 & 0 & 52 \\
SP 3 & 2 & 4 & 26 & 4 & 0 & 1 & 0 & 0 & 37 \\
SP 4 & 0 & 0 & 4 & 13 & 4 & 0 & 1 & 1 & 23 \\
SP 5 & 0 & 2 & 1 & 6 & 11 & 1 & 0 & 0 & 21 \\
SP 6 & 0 & 0 & 1 & 2 & 5 & 10 & 1 & 2 & 22 \\
SP 7 & 0 & 0 & 0 & 2 & 6 & 8 & 14 & 2 & 32 \\
SP 8 & 0 & 0 & 1 & 1 & 2 & 5 & 6 & 34 & 49 \\
Total & 75 & 54 & 36 & 28 & 30 & 25 & 24 & 39 & 312 \\
& & & & Short Words & & & & \\
SP 1 & 122 & 1 & 0 & 0 & 0 & 0 & 0 & 0 & 123 \\
SP 2 & 3 & 103 & 1 & 0 & 0 & 0 & 0 & 0 & 107 \\
SP 3 & 0 & 3 & 79 & 2 & 1 & 0 & 0 & 0 & 85 \\
SP 4 & 0 & 0 & 9 & 50 & 2 & 1 & 0 & 0 & 62 \\
SP 5 & 0 & 1 & 3 & 8 & 30 & 3 & 2 & 1 & 48 \\
SP 6 & 0 & 1 & 1 & 4 & 12 & 17 & 3 & 0 & 38 \\
SP 7 & 0 & 0 & 1 & 3 & 3 & 12 & 22 & 6 & 47 \\
SP 8 & 0 & 2 & 1 & 1 & 0 & 4 & 10 & 23 & 41 \\
Total & 125 & 111 & 95 & 68 & 48 & 37 & 37 & 30 & 551 \\
\hline
\end{tabular}

Table 3D

Analysis of Output Order in Experiment 3: Number of Words Recalled at Each Output Position As a Function of Experimental Task, Word Length, and Serial Position in the Free Recall Quiet Condition

\begin{tabular}{|c|c|c|c|c|c|c|c|c|c|}
\hline \multirow{2}{*}{$\begin{array}{c}\text { Serial } \\
\text { Position } \\
\end{array}$} & \multicolumn{8}{|c|}{ Output Position } & \multirow[b]{2}{*}{ Total } \\
\hline & 1 & 2 & 3 & 4 & 5 & 6 & 7 & 8 & \\
\hline \multicolumn{10}{|c|}{ Long Words } \\
\hline SP 1 & 7 & 14 & 7 & 11 & 6 & 0 & 0 & 0 & 45 \\
\hline SP 2 & 6 & 9 & 12 & 9 & 2 & 0 & 0 & 0 & 38 \\
\hline SP 3 & 3 & 8 & 10 & 1 & 3 & 1 & 0 & 0 & 26 \\
\hline SP 4 & 5 & 6 & 11 & 4 & 2 & 0 & 0 & 0 & 28 \\
\hline SP 5 & 0 & 8 & 17 & 4 & 1 & 0 & 0 & 0 & 30 \\
\hline SP 6 & 6 & 17 & 14 & 5 & 0 & 1 & 0 & 0 & 43 \\
\hline SP 7 & 17 & 43 & 7 & 7 & 0 & 0 & 0 & 0 & 74 \\
\hline SP 8 & 92 & 22 & 9 & 1 & 0 & 0 & 0 & 0 & 124 \\
\hline Total & 136 & 127 & 87 & 42 & 14 & 2 & 0 & 0 & 408 \\
\hline \multicolumn{10}{|c|}{ Short Words } \\
\hline SP 1 & 38 & 12 & 19 & 16 & 13 & 6 & 2 & 0 & 106 \\
\hline SP 2 & 6 & 39 & 13 & 13 & 15 & 7 & 3 & 0 & 96 \\
\hline SP 3 & 1 & 3 & 24 & 15 & 14 & 8 & 10 & 0 & 75 \\
\hline SP 4 & 2 & 8 & 11 & 21 & 6 & 6 & 2 & 2 & 58 \\
\hline SP 5 & 17 & 6 & 10 & 15 & 11 & 5 & 0 & 1 & 65 \\
\hline SP 6 & 12 & 18 & 18 & 10 & 7 & 2 & 1 & 0 & 68 \\
\hline SP 7 & 14 & 32 & 19 & 8 & 6 & 2 & 2 & 2 & 85 \\
\hline SP 8 & 49 & 22 & 19 & 10 & 5 & 4 & 0 & 0 & 109 \\
\hline Total & 139 & 140 & 133 & 108 & 77 & 40 & 20 & 5 & 662 \\
\hline
\end{tabular}

in Experiment 3, tabulated by the output order and the serial position of the words in the list. Again, the participants who were required to perform ISR initiated their recall with the first word in the list and tended to proceed forward in their recall. Their ability to access the first list items was greater for the short words than for the long words and was also greater in the quiet conditions than in 
the articulatory suppression conditions. The participants who were required to perform free recall tended to initiate their recall from toward the end of the list. However, on a minority of trials, there was also a tendency for the first word in the list to be recalled first in the free recall conditions. The size of this minority tendency was greater for the short words than for the long words and was also greater in the quiet conditions than in the articulatory suppression conditions. As in the ISR conditions, this tendency to initiate free recall with the first word in the list was greater for the short words than for the long words and was also greater in the quiet conditions than in the articulatory suppression conditions.

\section{Discussion}

Experiment 3 confirmed and extended the results of Experiment 2. First, there were again different-shaped serial position curves for the two tasks, with free recall characterized by recency and primacy (for lists of short words) and ISR characterized by extended primacy and little or no recency.

Second, there were word length effects and effects of articulatory suppression for both free recall and ISR. In both tasks, the effects of word length were greatest at the early serial positions and were reduced but not eliminated by articulatory suppression. Although word length effects are often eliminated in ISR when the stimuli are presented visually under articulatory suppression (Baddeley et al., 1984; Baddeley et al., 1975; Longoni et al., 1993), this finding seems to be obtained when participants are presented with stimuli from a restricted stimulus set. When the stimuli are selected from a large stimulus set, as was the case in this study, it is more common to experience a reduction, rather than an elimination, of the word length effect following suppression (La Pointe \& Engle, 1990; Russo \& Grammatopoulou, 2003).

The findings of word length effects and effects of articulatory suppression in free recall replicate the results of previous studies of word length effects in free recall (Russo \& Grammatopoulou, 2003; Salthouse, 1980; Watkins, 1972) and articulatory suppression effects in free recall (Richardson \& Baddeley, 1975; Russo \& Grammatopoulou, 2003) and demonstrate that similar findings are obtained for both free recall and ISR under identical methods of presentation.

\section{EXPERIMENT 4}

One possible limitation of Experiments 1-3 is that they used exclusively 8 -item lists. We chose list lengths of 8 items in Experiments 1-3 because we wanted to equate the methodology used in the two tasks as much as possible, and we thought that a single list length of 8 items provided a compromise between the typically shorter list lengths used in ISR and the typically longer list lengths used in free recall. However, one could argue that a compromise list length of 8 is neither fully representative of the longer list lengths typically used in free recall (e.g., 10-40 items; Murdock, 1962) nor fully representative of the shorter list lengths typically used in ISR (e.g., 5-6 items; Baddeley, 1986).

One particularly worrying aspect about using longer than normal ISR lists is that some researchers have claimed that only ISR data collected with shorter lists (typically five or six items) might make use of specialized short-term or working memory mechanisms (e.g., Baddeley, 2007; Baddeley \& Larsen, 2003, 2007; Campoy \& Baddeley, 2008; Larsen \& Baddeley, 2003). Hence, it was decided to reexamine the basic word length effect obtained in the two tasks in Experiments 2 and 3 using shorter lists of six items.

\section{Method}

Participants. Twenty students from the University of Essex participated in this experiment. None had taken part in any of the previous experiments.

Materials. The participants saw a total of 36 lists of six words that were randomly allocated without replacement for each participant from the same three sets of words used in Experiment 2.

Design. The experiment used a totally within-subjects design, with two independent variables: word length, with three levels (onesyllable, three-syllable, and five-syllable words), and serial position, with six levels (SPs 1-6). Each participant received trials using both the free recall task and the ISR task.

Procedure. The participants were tested individually and were informed that they would be shown 2 practice lists of six words, followed by 36 experimental trials of six words. The experimental trials were divided into two blocks of 18 experimental trials. The participants were tested using free recall in one block and ISR in the other, and the order of the two blocks was counterbalanced across participants. The participants were instructed as to which task to perform prior to the presentation of stimuli of each block. Within the 18 trials of each block, there were 6 trials of six short words, 6 trials of six medium words, and 6 trials of six long words. The 18 lists were presented in a random order within each block. On all the trials, the participants were instructed to read aloud each word as it was presented on the screen, but they were not required to rehearse out loud. Each trial began with a warning tone, followed after $1 \mathrm{sec}$ by a series of six words. The words were presented individually in the middle of the computer screen for $2 \mathrm{sec}$ per word. After the last word from the study list had been presented, a series of beeps signaled the beginning of a $20-\mathrm{sec}$ recall period for verbal recall. A tape recorder was used to record the participants' recall performance.

\section{Results}

Free recall. The proportions of items recalled at each serial position for each word length and articulation condition in free recall are shown in Figure 7A. A $3 \times 6$ fully within-subjects ANOVA with two factors-word length, with three levels (short, medium, or long), and serial position, with six levels (1-6) - revealed a significant main effect of word length $\left[F(2,19)=80.44, M S_{\mathrm{e}}=\right.$ $0.026, p<.0001]$, a significant main effect of serial position $\left[F(5,95)=37.50, M S_{\mathrm{e}}=0.058, p<.0001\right]$, and a significant word length $\times$ serial position interaction $\left[F(10,190)=7.20, M S_{\mathrm{e}}=0.028, p<.0001\right]$. An analysis of the main effect of word length, using Newman-Keuls pairwise comparisons, revealed that the recall performance for each word length was significantly different from that for all the others.

A simple main effects analysis of the two-way interaction showed that the simple main effect of word length was sig- 
A Experiment 4: Free Recall



B

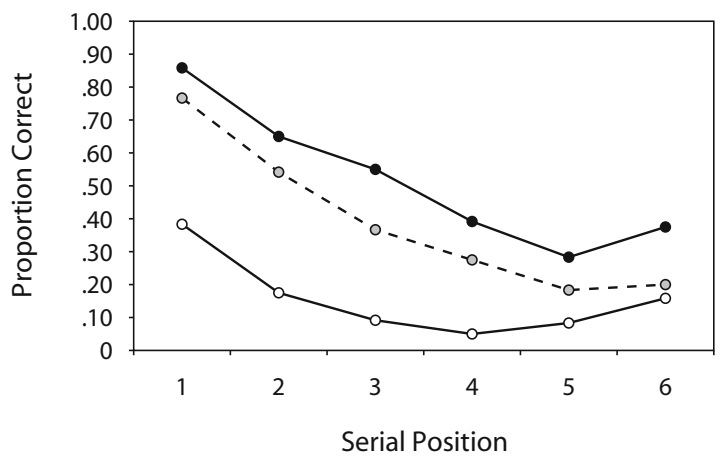

Figure 7. Experiment 4: Serial position curves for the lists of short, medium, and long words presented for free recall (A) and immediate serial recall (ISR) (B).

nificant only at the first four serial positions. There were also significant recency effects in all conditions, but the most extended recency effects were with the longer word lengths.

ISR. The proportions of items recalled in their correct serial position for each word length in ISR are illustrated in Figure 7B. A $3 \times 6$ fully within-subjects ANOVA with two factors - word length, with three levels (short, medium, or long), and serial position, with six levels (1-6) - revealed a significant main effect of word length $[F(2,19)=45.46$, $\left.M S_{\mathrm{e}}=0.088, p<.0001\right]$, a significant main effect of serial position $\left[F(5,95)=55.18, M S_{\mathrm{e}}=0.036, p<.0001\right]$, and a significant word length $\times$ serial position interaction $\left[F(10,190)=5.44, M S_{\mathrm{e}}=0.023, p<.0001\right]$. An analysis of the main effect of word length, using Newman-Keuls pairwise comparisons, revealed that the recall performance of each word length was significantly different from that for all the others.

A simple main effects analysis of the two-way interaction showed that the simple main effect of word length was significant only at the first four serial positions. There were also significant recency effects in all the conditions, but the most extended recency effects were with the longer word lengths.
Analysis of output order. Tables 4A and 4B show the count of the words recalled in all the six different conditions in Experiment 4, tabulated by the output order and the serial position of the words in the list. The participants who were required to perform ISR initiated their recall with the first word in the list and tended to proceed forward in their recall. Their ability to access the first list item decreased with increasing word length. By contrast, those who were required to perform free recall tended to initiate their recall toward the end of the list. However, there was also a tendency to initiate free recall with the first word in the list, and as for ISR, this tendency decreased with increasing word length.

\section{Discussion}

Experiment 4 showed clear word length effects for both free recall and ISR, using lists of six words. Reassuringly, our results generalized the word length effects obtained in both tasks in Experiments 2 and 3 to shorter lists of six words, a length for which some researchers hypothesize that our participants might have made use of specialized short-term or working memory mechanisms (e.g., Baddeley \& Larsen, 2003; Larsen \& Baddeley, 2003).

\section{EXPERIMENT 5}

The aim of Experiment 5 was also to replicate the word length effects in free recall and ISR, but this time using lists that were longer than eight words and, hence, more typical of those used in free recall. Although, to our knowledge, no theory of free recall has ever been proposed that was limited to explaining longer lists but not shorter lists, it is the case that list length in free recall affects output order. Recent, and as yet unpublished, work shows that participants who are presented with very short lists of words (1-4) for free recall tend to initiate their output from the very beginning of the list in forward order, whereas with longer lists $(8+)$, they tend to output the most recent items first (Ward, Tan, \& Grenfell-Essam, 2009).

It would be reassuring, therefore, to see whether the word length effects obtained in free recall and ISR in Experiments 1-4 could also be observed in Experiment 5, which tested free recall and ISR with lists of 12 items. We anticipated that the participants would have difficulty in performing ISR with 12-item lists, and so we adopted the task instructions for ISR used recently by Golomb et al. (2008), who tested ISR with 10 -item lists. These instructions explicitly encouraged participants to begin recall with the very first list item, but if this was not possible, they were instructed to begin recall with the earliest item that they could remember. The participants were not required to make exactly 12 responses or say "blank," but they were instructed to continue their recall as far as possible in a forward serial order.

\section{Method}

Participants. Twenty students from the University of Essex participated in this experiment. None had taken part in any of the previous experiments. 
Table 4A

Analysis of Output Order in Experiment 4: Number of Words Recalled at Each Output Position As a Function of Experimental Task, Word Length, and Serial Position in the Immediate Serial Recall Task

\begin{tabular}{|c|c|c|c|c|c|c|c|}
\hline \multirow{2}{*}{$\begin{array}{c}\text { Serial } \\
\text { Position }\end{array}$} & \multicolumn{6}{|c|}{ Output Position } & \multirow[b]{2}{*}{ Total } \\
\hline & 1 & 2 & 3 & 4 & 5 & 6 & \\
\hline \multicolumn{8}{|c|}{ Long Words } \\
\hline SP 1 & 46 & 2 & 3 & 2 & 2 & 1 & 56 \\
\hline SP 2 & 7 & 21 & 6 & 3 & 1 & 0 & 38 \\
\hline SP 3 & 9 & 11 & 11 & 3 & 0 & 0 & 34 \\
\hline SP 4 & 3 & 5 & 10 & 6 & 3 & 0 & 27 \\
\hline SP 5 & 6 & 14 & 5 & 6 & 10 & 3 & 44 \\
\hline SP 6 & 10 & 10 & 11 & 2 & 2 & 19 & 54 \\
\hline Total & 81 & 63 & 46 & 22 & 18 & 23 & 253 \\
\hline \multicolumn{8}{|c|}{ Medium Words } \\
\hline SP 1 & 92 & 4 & 2 & 1 & 1 & 0 & 100 \\
\hline SP 2 & 5 & 65 & 2 & 3 & 0 & 0 & 75 \\
\hline SP 3 & 6 & 11 & 44 & 4 & 2 & 0 & 67 \\
\hline SP 4 & 2 & 8 & 8 & 33 & 6 & 1 & 58 \\
\hline SP 5 & 1 & 8 & 22 & 13 & 22 & 3 & 69 \\
\hline SP 6 & 2 & 4 & 13 & 13 & 16 & 24 & 72 \\
\hline Total & 108 & 100 & 91 & 67 & 47 & 28 & 441 \\
\hline \multicolumn{8}{|c|}{ Short Words } \\
\hline SP 1 & 103 & 0 & 1 & 3 & 1 & 0 & 108 \\
\hline SP 2 & 1 & 78 & 2 & 1 & 1 & 0 & 83 \\
\hline SP 3 & 0 & 5 & 66 & 5 & 2 & 1 & 79 \\
\hline SP 4 & 1 & 5 & 10 & 47 & 4 & 2 & 69 \\
\hline SP 5 & 1 & 4 & 8 & 14 & 34 & 1 & 62 \\
\hline SP 6 & 2 & 1 & 6 & 11 & 13 & 45 & 79 \\
\hline Total & 108 & 93 & 93 & 81 & 55 & 49 & 480 \\
\hline
\end{tabular}

Materials. The participants saw a total of 36 lists of 12 words that were randomly allocated without replacement for each participant from the same three sets of words as those used in Experiment 2.

Design. The experiment used a totally within-subjects design, with two independent variables: word length, with 3 levels (onesyllable, three-syllable, and five-syllable words), and serial position, with 12 levels (SPs 1-12). Each participant received trials using both the free recall task and the ISR task.

Procedure. The participants were tested individually and were informed that they would be shown 2 practice lists of 12 words, followed by 36 experimental trials of 12 words. The experimental trials were divided into two blocks of 18 experimental trials. The participants were tested using free recall in one block and ISR in the other, and the order of the two blocks was counterbalanced across participants. The participants were instructed as to which task to perform prior to the presentation of the stimuli in each block. Within the 18 trials of each block, there were 6 trials of 6 short words, 6 trials of 6 medium words, and 6 trials of 6 long words. The 18 lists were presented in a random order within each block. On all the trials, the participants were instructed to read aloud each word as it was presented on the screen, but they were not required to rehearse out loud. Each trial began with a warning tone, followed after $1 \mathrm{sec}$ by a series of 6 words. The words were presented individually in the middle of the computer screen for $2 \mathrm{sec}$ per word. After the last word from the study list had been presented, a series of beeps signaled the beginning of the recall period for verbal recall. The recall period was not of a fixed length; rather, the participants pressed a computer button when they were ready to continue to the next trial. A tape recorder was used to record the participants' recall performance.

\section{Results}

Free recall. The proportions of items recalled at each serial position for each word length and articulation condition
Table 4B

Analysis of Output Order in Experiment 4: Number of Words Recalled at Each Output Position As a Function of Experimental Task, Word Length, and Serial Position in the Free Recall Task

\begin{tabular}{|c|c|c|c|c|c|c|c|}
\hline \multirow{2}{*}{$\begin{array}{c}\text { Serial } \\
\text { Position }\end{array}$} & \multicolumn{6}{|c|}{ Output Position } & \multirow[b]{2}{*}{ Total } \\
\hline & 1 & 2 & 3 & 4 & 5 & 6 & \\
\hline \multicolumn{8}{|c|}{ Long Words } \\
\hline SP 1 & 7 & 4 & 11 & 6 & 1 & 0 & 29 \\
\hline SP 2 & 7 & 3 & 9 & 5 & 3 & 0 & 27 \\
\hline SP 3 & 3 & 5 & 19 & 9 & 2 & 0 & 38 \\
\hline SP 4 & 7 & 12 & 14 & 9 & 1 & 1 & 44 \\
\hline SP 5 & 22 & 52 & 12 & 3 & 1 & 0 & 90 \\
\hline SP 6 & 67 & 28 & 11 & 1 & 0 & 0 & 107 \\
\hline Total & 113 & 104 & 76 & 33 & 8 & 1 & 335 \\
\hline \multicolumn{8}{|c|}{ Medium Words } \\
\hline SP 1 & 25 & 1 & 16 & 17 & 8 & 2 & 69 \\
\hline SP 2 & 1 & 26 & 13 & 18 & 10 & 1 & 69 \\
\hline SP 3 & 8 & 2 & 24 & 15 & 5 & 3 & 57 \\
\hline SP 4 & 8 & 19 & 28 & 14 & 1 & 0 & 70 \\
\hline SP 5 & 15 & 47 & 20 & 8 & 6 & 0 & 96 \\
\hline SP 6 & 63 & 21 & 8 & 9 & 11 & 4 & 116 \\
\hline Total & 120 & 116 & 109 & 81 & 41 & 10 & 477 \\
\hline \multicolumn{8}{|c|}{ Short Words } \\
\hline SP 1 & 47 & 6 & 15 & 12 & 6 & 2 & 88 \\
\hline SP 2 & 1 & 43 & 8 & 16 & 6 & 2 & 76 \\
\hline SP 3 & 5 & 10 & 30 & 11 & 9 & 3 & 68 \\
\hline SP 4 & 8 & 16 & 23 & 27 & 7 & 2 & 83 \\
\hline SP 5 & 13 & 33 & 20 & 11 & 13 & 1 & 91 \\
\hline SP 6 & 45 & 11 & 18 & 14 & 8 & 14 & 111 \\
\hline Total & 119 & 119 & 114 & 91 & 49 & 24 & 517 \\
\hline
\end{tabular}

in free recall are shown in Figure 8A. A $3 \times 12$ fully withinsubjects ANOVA with two factors - word length (short, medium, or long) and serial position (1-12) - revealed a significant main effect of word length $[F(2,38)=28.62$, $\left.M S_{\mathrm{e}}=0.031, p<.0001\right]$, a significant main effect of serial position $\left[F(11,209)=122.0, M S_{\mathrm{e}}=0.033, p<.0001\right]$, and a significant word length $\times$ serial position interaction $\left[F(22,418)=2.25, M S_{\mathrm{e}}=0.023, p<.005\right]$.

An analysis of the main effect of word length, using Newman-Keuls pairwise comparisons, revealed that the recall performance for each word length was significantly different from that for all the others. A simple main effects analysis of the two-way interaction showed that the simple main effect of word length was significant at SP 2 and at SPs $8-11$. There were also significant recency effects in all three word length conditions.

ISR (standard ISR scoring). ISR performance was first examined under standard ISR scoring, in which an item is marked correct only if it was output in the correct output position (i.e., the first item in the list must be recalled first, the second item recalled second, and so on). The proportions of items recalled at each serial position for each word length and articulation condition in ISR are shown in Figure 8B. A $3 \times 12$ fully withinsubjects ANOVA with two factors - word length (short, medium, or long) and serial position (1-12) - revealed a significant main effect of word length $[F(2,38)=5.112$, $\left.M S_{\mathrm{e}}=0.026, p<.05\right]$, a significant main effect of serial position $\left[F(11,209)=13.98, M S_{\mathrm{e}}=0.026, p<.0001\right]$, 
A

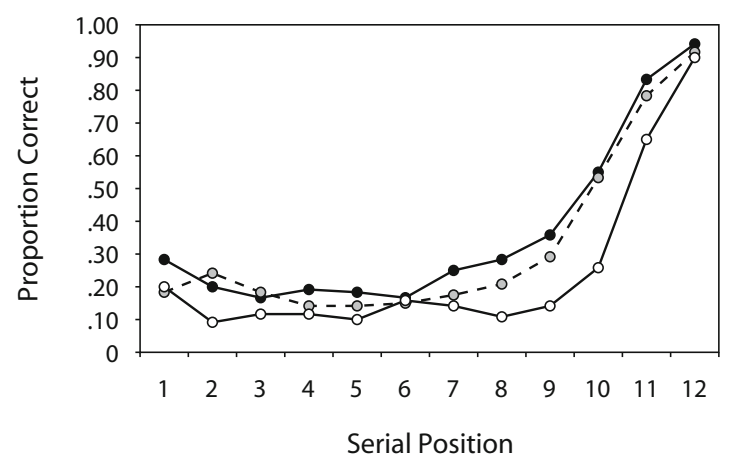

C Experiment 5: ISR (Exact Relative Scoring)

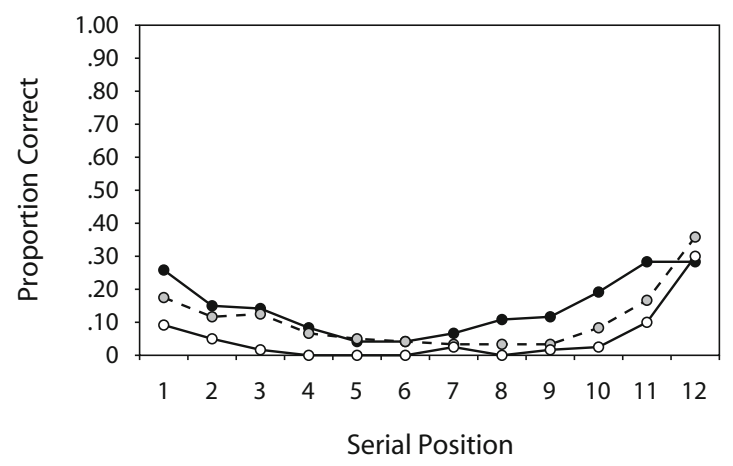

B Experiment 5: ISR (Standard Scoring)

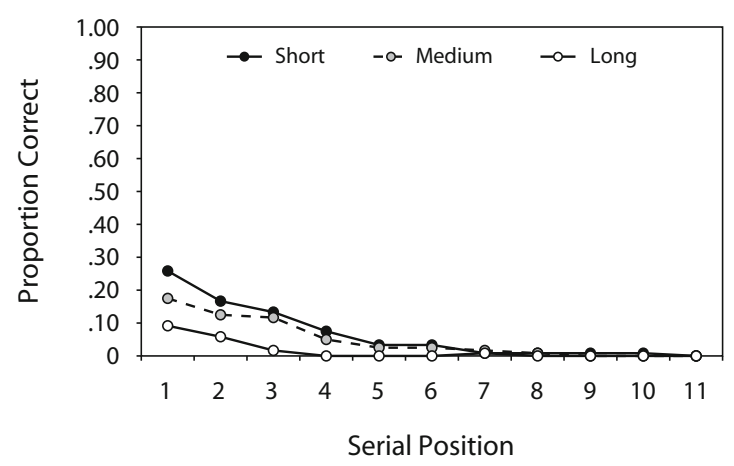

D

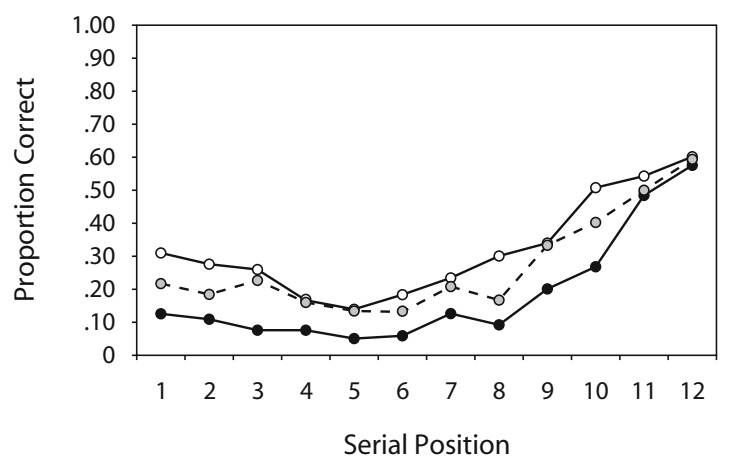

Figure 8. Experiment 5: (A) Serial position curves for the lists of short, medium, and long words presented for free recall. (B) Serial position curves for the lists of short, medium, and long words scored for immediate serial recall (ISR), using the standard ISR scoring, in which a word is considered correct only if it is output in its correct absolute position. (C) Serial position curves for the ISR data using the exact relative scoring measure. If one represents a 12-item list as "A B C D E F G H I J K L," Word A is considered correct if it is output first, and any other word is considered correct if it is output immediately after its immediate predecessor in the list (e.g., in the output "A C $\underline{\mathrm{D}} \underline{\mathrm{E}} \mathrm{G}$ F J K $\underline{\mathrm{L}}$," only the underlined items at Serial Positions 1, 4, 5, 11, and 12 would be deemed to be recalled correctly in the exact relative order, because $A$ is output first, and $D$ is recalled immediately after $C, E$ after $D, K$ after $J$, and $L$ after $K$ ).

and a significant word length $\times$ serial position interaction $\left[F(22,418)=3.51, M S_{\mathrm{e}}=0.005, p<.005\right]$. An analysis of the main effect of word length, using Newman-Keuls pairwise comparisons, revealed that the recall performance for the long words differed from that for the medium and short words, but there was no significant difference between the short words and the medium words.

A simple main effects analysis of the two-way interaction showed that the simple main effect of word length was significant only at SP $1(p<.01)$ and was close to significance at SP $3(p<.06)$. There were also significant primacy effects for the two shorter length words.

ISR (exact relative order scoring). ISR performance was next examined under exact relative order scoring, in which items were marked correct only if words that were successively presented at input were output together in immediate succession at recall. If the 12 letters in the sequence "ABCDEFGHIJKL" represent the 12 words presented at input, the exact relative ordering scoring system scores A in the correct position if it is output first, B in the correct relative order if it is output immediately after A, and so on, so that $\mathrm{L}$ will be assumed to be output in the correct relative order if it was output immediately after $\mathrm{K}$. The proportions of items recalled correctly using this exact relative ordering scoring at each serial position for each word length and articulation condition in ISR are shown in Figure 8C. A $3 \times 12$ fully within-subjects ANOVA with two factors - word length (short, medium, or long) and serial position (1-12) - revealed a significant main effect of word length $\left[F(2,38)=13.17, M S_{\mathrm{e}}=0.042, p<.0001\right]$, a significant main effect of serial position $[F(11,209)=$ 17.21, $\left.M S_{\mathrm{e}}=0.025, p<.0001\right]$, and a significant word length $\times$ serial position interaction $[F(22,418)=2.071$, $\left.M S_{\mathrm{e}}=0.012, p<.01\right]$. An analysis of the main effect of word length, using Newman-Keuls pairwise comparisons, revealed that there were significant differences between all different pairs of word lengths.

A simple main effects analysis of the two-way interaction showed that the simple main effect of word length was significant only at SP $1(p<.01)$ and was close to 
significance at SP $3(p<.06)$. There were also significant primacy effects for the two shorter length words and significant recency effects for all word lengths.

ISR (free recall scoring). ISR performance was finally examined using free recall scoring. The proportions of items recalled at each serial position for each word length and articulation condition in ISR are shown in Figure 8D. A $3 \times 12$ fully within-subjects ANOVA with two factorsword length (short, medium, or long) and serial position (1-12) - revealed a significant main effect of word length $\left[F(2,38)=31.83, M S_{\mathrm{e}}=0.035, p<.0001\right]$, a significant main effect of serial position $\left[F(11,209)=28.87, M S_{\mathrm{e}}=\right.$ $0.051, p<.0001]$, and a nonsignificant word length $\times$ serial position interaction $\left[F(22,418)<1, M S_{\mathrm{e}}=0.028\right.$, $p>.05]$. An analysis of the main effect of word length, using Newman-Keuls pairwise comparisons-revealed that there were significant differences between all different pairs of word lengths. Analyses on all three serial position curves revealed significant and extended recency effects but no primacy effects.

Analysis of output order. We examined the order in which the words were output at recall in the six different conditions. Tables 5A and 5B show the count of all the words recalled in the six conditions in Experiment 5, tabulated by the output order and the serial position of the words in the list. With 12-item lists, the participants found it difficult to initiate their recall with the first word in the list. In ISR, recall initiated with the first item on a small minority of trials, and this tendency increased for shorter words. There was no tendency for the participants in the

Table 5A

Analysis of Output Order in Experiment 5: Number of Words Recalled at Each Output Position As a Function of Experimental Task, Word Length, and Serial Position in the Immediate Serial Recall Task

\begin{tabular}{|c|c|c|c|c|c|c|c|c|c|c|c|c|}
\hline \multirow{2}{*}{$\begin{array}{c}\text { Serial } \\
\text { Position }\end{array}$} & \multicolumn{11}{|c|}{ Output Position } & \multirow[b]{2}{*}{ Total } \\
\hline & 1 & 2 & 3 & 4 & 5 & 6 & 7 & 8 & 9 & 10 & 11 & \\
\hline \multicolumn{13}{|c|}{ Long Words } \\
\hline SP 1 & 11 & 3 & 1 & 0 & 0 & 0 & 0 & 0 & 0 & 0 & 0 & 15 \\
\hline SP 2 & 5 & 7 & 1 & 0 & 0 & 0 & 0 & 0 & 0 & 0 & 0 & 13 \\
\hline SP 3 & 2 & 3 & 2 & 2 & 0 & 0 & 0 & 0 & 0 & 0 & 0 & 9 \\
\hline SP 4 & 5 & 0 & 4 & 0 & 0 & 0 & 0 & 0 & 0 & 0 & 0 & 9 \\
\hline SP 5 & 3 & 2 & 1 & 0 & 0 & 0 & 0 & 0 & 0 & 0 & 0 & 6 \\
\hline SP 6 & 3 & 1 & 2 & 1 & 0 & 0 & 0 & 0 & 0 & 0 & 0 & 7 \\
\hline SP 7 & 6 & 4 & 1 & 3 & 0 & 0 & 1 & 0 & 0 & 0 & 0 & 15 \\
\hline SP 8 & 6 & 2 & 2 & 1 & 0 & 0 & 0 & 0 & 0 & 0 & 0 & 11 \\
\hline SP 9 & 12 & 6 & 3 & 1 & 1 & 1 & 0 & 0 & 0 & 0 & 0 & 24 \\
\hline SP 10 & 18 & 6 & 5 & 1 & 1 & 0 & 0 & 1 & 0 & 0 & 0 & 32 \\
\hline SP 11 & 22 & 24 & 4 & 5 & 2 & 1 & 0 & 0 & 0 & 0 & 0 & 58 \\
\hline SP 12 & 4 & 32 & 29 & 4 & 0 & 0 & 0 & 0 & 0 & 0 & 0 & 69 \\
\hline Total & 97 & 90 & 55 & 18 & 4 & 2 & 1 & 1 & 0 & 0 & 0 & 268 \\
\hline \multicolumn{13}{|c|}{ Medium Words } \\
\hline SP 1 & 21 & 2 & 2 & 0 & 1 & 0 & 0 & 0 & 0 & 0 & 0 & 26 \\
\hline SP 2 & 6 & 15 & 0 & 0 & 1 & 0 & 0 & 0 & 0 & 0 & 0 & 22 \\
\hline SP 3 & 7 & 4 & 14 & 2 & 0 & 0 & 0 & 0 & 0 & 0 & 0 & 27 \\
\hline SP 4 & 5 & 3 & 5 & 6 & 0 & 0 & 0 & 0 & 0 & 0 & 0 & 19 \\
\hline SP 5 & 3 & 1 & 3 & 6 & 3 & 0 & 0 & 0 & 0 & 0 & 0 & 16 \\
\hline SP 6 & 8 & 3 & 0 & 1 & 1 & 3 & 0 & 0 & 0 & 0 & 0 & 16 \\
\hline SP 7 & 8 & 6 & 4 & 1 & 2 & 2 & 2 & 0 & 0 & 0 & 0 & 25 \\
\hline SP 8 & 4 & 7 & 3 & 1 & 2 & 2 & 0 & 1 & 0 & 0 & 0 & 20 \\
\hline SP 9 & 17 & 8 & 7 & 4 & 3 & 0 & 1 & 0 & 0 & 0 & 0 & 40 \\
\hline SP 10 & 18 & 16 & 6 & 4 & 2 & 1 & 0 & 1 & 0 & 0 & 0 & 48 \\
\hline SP 11 & 8 & 25 & 14 & 6 & 4 & 0 & 0 & 1 & 2 & 0 & 0 & 60 \\
\hline SP 12 & 0 & 11 & 26 & 23 & 7 & 2 & 0 & 0 & 0 & 2 & 0 & 71 \\
\hline Total & 105 & 101 & 84 & 54 & 26 & 10 & 3 & 3 & 2 & 2 & 0 & 390 \\
\hline \multicolumn{13}{|c|}{ Short Words } \\
\hline SP 1 & 31 & 1 & 2 & 2 & 1 & 0 & 0 & 0 & 0 & 0 & 0 & 37 \\
\hline SP 2 & 6 & 20 & 5 & 1 & 1 & 0 & 0 & 0 & 0 & 0 & 0 & 33 \\
\hline SP 3 & 5 & 9 & 16 & 0 & 1 & 0 & 0 & 0 & 0 & 0 & 0 & 31 \\
\hline SP 4 & 3 & 3 & 4 & 9 & 1 & 0 & 0 & 0 & 0 & 0 & 0 & 20 \\
\hline SP 5 & 4 & 1 & 5 & 3 & 4 & 0 & 0 & 0 & 0 & 0 & 0 & 17 \\
\hline SP 6 & 8 & 2 & 2 & 4 & 2 & 4 & 0 & 0 & 0 & 0 & 0 & 22 \\
\hline SP 7 & 8 & 8 & 2 & 5 & 3 & 1 & 1 & 0 & 0 & 0 & 0 & 28 \\
\hline SP 8 & 11 & 10 & 2 & 6 & 2 & 2 & 2 & 1 & 0 & 0 & 0 & 36 \\
\hline SP 9 & 13 & 16 & 5 & 2 & 1 & 1 & 1 & 1 & 1 & 0 & 0 & 41 \\
\hline SP 10 & 15 & 15 & 15 & 9 & 0 & 3 & 0 & 2 & 1 & 1 & 0 & 61 \\
\hline SP 11 & 6 & 16 & 22 & 8 & 6 & 2 & 2 & 0 & 2 & 1 & 0 & 65 \\
\hline SP 12 & 2 & 7 & 20 & 24 & 10 & 4 & 3 & 1 & 0 & 1 & 0 & 72 \\
\hline Total & 112 & 108 & 100 & 73 & 32 & 17 & 9 & 5 & 4 & 3 & 0 & 463 \\
\hline
\end{tabular}


Table 5B

Analysis of Output Order in Experiment 5: Number of Words Recalled at Each Output Position As a Function of Experimental Task, Word Length, and Serial Position in the Free Recall Task

\begin{tabular}{|c|c|c|c|c|c|c|c|c|c|c|c|c|}
\hline \multirow{2}{*}{$\begin{array}{c}\text { Serial } \\
\text { Position } \\
\end{array}$} & \multicolumn{11}{|c|}{ Output Position } & \multirow[b]{2}{*}{ Tota } \\
\hline & 1 & 2 & 3 & 4 & 5 & 6 & 7 & 8 & 9 & 10 & 11 & \\
\hline \multicolumn{13}{|c|}{ Long Words } \\
\hline SP 1 & 2 & 4 & 7 & 8 & 2 & 1 & 0 & 0 & 0 & 0 & 0 & 24 \\
\hline SP 2 & 0 & 4 & 4 & 2 & 1 & 0 & 0 & 0 & 0 & 0 & 0 & 11 \\
\hline SP 3 & 0 & 0 & 4 & 6 & 2 & 2 & 0 & 0 & 0 & 0 & 0 & 14 \\
\hline SP 4 & 2 & 0 & 2 & 6 & 4 & 0 & 0 & 0 & 0 & 0 & 0 & 14 \\
\hline SP 5 & 0 & 2 & 4 & 4 & 1 & 0 & 1 & 0 & 0 & 0 & 0 & 12 \\
\hline SP 6 & 0 & 3 & 3 & 5 & 5 & 2 & 0 & 1 & 0 & 0 & 0 & 19 \\
\hline SP 7 & 0 & 1 & 8 & 5 & 3 & 0 & 0 & 0 & 0 & 0 & 0 & 17 \\
\hline SP 8 & 1 & 2 & 5 & 2 & 1 & 1 & 1 & 0 & 0 & 0 & 0 & 13 \\
\hline SP 9 & 0 & 2 & 10 & 1 & 3 & 1 & 0 & 0 & 0 & 0 & 0 & 17 \\
\hline SP 10 & 8 & 10 & 12 & 1 & 0 & 0 & 0 & 0 & 0 & 0 & 0 & 31 \\
\hline SP 11 & 15 & 51 & 11 & 1 & 0 & 0 & 0 & 0 & 0 & 0 & 0 & 78 \\
\hline SP 12 & 84 & 22 & 2 & 0 & 0 & 0 & 0 & 0 & 0 & 0 & 0 & 108 \\
\hline Total & 112 & 101 & 72 & 41 & 22 & 7 & 2 & 1 & 0 & 0 & 0 & 358 \\
\hline \multicolumn{13}{|c|}{ Medium Words } \\
\hline SP 1 & 4 & 4 & 1 & 5 & 4 & 1 & 1 & 1 & 1 & 0 & 0 & 22 \\
\hline SP 2 & 1 & 4 & 7 & 7 & 3 & 4 & 0 & 3 & 0 & 0 & 0 & 29 \\
\hline SP 3 & 2 & 1 & 1 & 7 & 7 & 2 & 2 & 0 & 0 & 0 & 0 & 22 \\
\hline SP 4 & 1 & 1 & 4 & 5 & 4 & 1 & 0 & 0 & 1 & 0 & 0 & 17 \\
\hline SP 5 & 1 & 1 & 1 & 4 & 4 & 2 & 4 & 0 & 0 & 0 & 0 & 17 \\
\hline SP 6 & 0 & 2 & 7 & 3 & 2 & 3 & 0 & 0 & 1 & 0 & 0 & 18 \\
\hline SP 7 & 1 & 2 & 4 & 8 & 3 & 1 & 0 & 1 & 0 & 0 & 1 & 21 \\
\hline SP 8 & 1 & 4 & 8 & 5 & 3 & 3 & 1 & 0 & 0 & 0 & 0 & 25 \\
\hline SP 9 & 4 & 3 & 11 & 12 & 3 & 0 & 1 & 1 & 0 & 0 & 0 & 35 \\
\hline SP 10 & 8 & 16 & 27 & 7 & 3 & 1 & 1 & 1 & 0 & 0 & 0 & 64 \\
\hline SP 11 & 17 & 61 & 15 & 0 & 1 & 0 & 0 & 0 & 0 & 0 & 0 & 94 \\
\hline SP 12 & 75 & 18 & 11 & 3 & 3 & 0 & 0 & 0 & 0 & 0 & 0 & 110 \\
\hline Total & 115 & 117 & 97 & 66 & 40 & 18 & 10 & 7 & 3 & 0 & 1 & 474 \\
\hline \multicolumn{13}{|c|}{ Short Words } \\
\hline SP 1 & 2 & 3 & 4 & 11 & 3 & 8 & 1 & 1 & 0 & 1 & 0 & 34 \\
\hline SP 2 & 1 & 3 & 3 & 4 & 4 & 5 & 2 & 0 & 1 & 1 & 0 & 24 \\
\hline SP 3 & 2 & 0 & 2 & 2 & 6 & 4 & 2 & 2 & 0 & 0 & 0 & 20 \\
\hline SP 4 & 0 & 1 & 2 & 6 & 4 & 4 & 4 & 1 & 1 & 0 & 0 & 23 \\
\hline SP 5 & 2 & 1 & 5 & 7 & 3 & 2 & 1 & 1 & 0 & 0 & 0 & 22 \\
\hline SP 6 & 3 & 0 & 3 & 5 & 5 & 1 & 1 & 1 & 0 & 1 & 0 & 20 \\
\hline SP 7 & 3 & 2 & 6 & 8 & 4 & 5 & 1 & 0 & 0 & 0 & 1 & 30 \\
\hline SP 8 & 0 & 4 & 9 & 10 & 7 & 0 & 2 & 0 & 2 & 0 & 0 & 34 \\
\hline SP 9 & 7 & 5 & 17 & 8 & 3 & 0 & 1 & 1 & 0 & 1 & 0 & 43 \\
\hline SP 10 & 15 & 11 & 23 & 8 & 7 & 2 & 0 & 0 & 0 & 0 & 0 & 66 \\
\hline SP 11 & 10 & 70 & 13 & 3 & 1 & 1 & 1 & 0 & 1 & 0 & 0 & 100 \\
\hline SP 12 & 73 & 13 & 19 & 7 & 1 & 0 & 0 & 0 & 0 & 0 & 0 & 113 \\
\hline Total & 118 & 113 & 106 & 79 & 48 & 32 & 16 & 7 & 5 & 4 & 1 & 529 \\
\hline
\end{tabular}

free recall conditions to initiate recall with the first list item. Rather, with these longer lists (and slightly different ISR instructions), the participants tended to output the later list items first.

\section{Discussion}

Experiment 5 showed word length effects for both free recall and ISR, using longer lists of 12 words. Consistent with the results of previous studies (Russo \& Grammatopoulou, 2003; Watkins, 1972), we obtained a significant word length effect in free recall for longer lists of 12 items. In our data, there was an interaction between word length and serial position: The word length effect reached significance at one early serial position and three late positions. A similar tendency for the word length effect to be great- est at early and late serial positions can also be observed in the Watkins data, although in that data set, the interaction between list half (SPs 1-6 and 7-12) and word length did not reach significance. The serial position curves of all word lengths were dominated by recency, and there was no significant primacy advantage for any word length.

As we anticipated, the participants found it difficult to perform the ISR task on 12-item lists. The participants rarely initiated recall with the first word in the list and then continued in strict serial order. When strict ISR scoring was used, there were primacy effects for the two shorter lists but poor overall performance. On the basis of limited data, there was some evidence of a word length effect, at least between the long words and the two shorter length words. 
However, there was a difference in the proportions of words recalled between all three word lengths in the ISR conditions when exact relative order scoring and free recall scoring systems were used. The majority of the words recalled in the ISR task were from the second half of the list, and recalling in relative serial order was most prevalent at the beginning and end of the list.

Thus, reassuringly, our main results generalize the word length effects obtained in both tasks in Experiments 2-4 to longer lists of 12 words.

\section{GENERAL DISCUSSION}

The aim of these experiments was to look for similarities and differences between the free recall and the ISR tasks by examining how the two tasks would be affected by factors linked directly or indirectly with rehearsal. Our motivation for the experiments was the current separation between theories and data concerned with ISR, on the one hand, and theories and data concerned with free recall, on the other (see also Ward, 2001; Ward et al., 2008). Although some accounts of memory do offer explanations of both tasks (e.g., Anderson et al., 1998; Brown et al., 2007), there are many accounts of free recall that have not, as yet, been applied to ISR (e.g., Davelaar et al., 2005; Gillund \& Shiffrin, 1984; Howard \& Kahana, 2002; Raaijmakers \& Shiffrin, 1981) and many accounts of ISR that have not, as yet, been applied to free recall (e.g., Baddeley, 1986, 2000, 2007; Brown et al., 2000; Burgess \& Hitch, 1992, 1999; Farrell \& Lewandowsky, 2002; Henson, 1998; Nairne, 1988; Page \& Norris, 1998).

We used the overt rehearsal technique and manipulations of presentation rate (taken from the free recall literature) and manipulations of word length and articulatory suppression (taken from the ISR literature) and applied all these techniques and manipulations to both tasks, testing under identical methodological conditions. To the extent to which we found similarities in the patterns of data, we would be tempted to consider that the two tasks shared common (or at least highly similar) rehearsal and recall processes.

\section{Observed Similarities Between Free Recall and ISR}

Two lines of evidence provide support for the hypothesis that participants make use of similar rehearsal and recall processes in free recall and ISR.

First, similar patterns of rehearsals were observed on the two tasks using the overt rehearsal method (Rundus \& Atkinson, 1970). In Experiment 1, the participants tended to rehearse the early words in the list more often than later words, in line with the classic overt rehearsal data in free recall by Rundus (1971) and more recent overt rehearsal data in ISR by Tan and Ward (2008). The rehearsal patterns were similar in both tasks, regardless of whether the task at test was precued or postcued. In Experiment 2, the recorded patterns of rehearsal were also similar across the free recall and ISR tasks: Rehearsal in both tasks was similarly reduced with longer words and fast presentation rates. These similar patterns of rehearsal in the two tasks are consistent with the existence of a common rehearsal process underpinning both free recall and ISR.

Second, similar effects on recall were observed in the two tasks by variables that are assumed to facilitate or hinder rehearsal. In Experiment 2, the word length and presentation rate were manipulated. There were significant effects of presentation rate and word length effects in both tasks. In Experiment 3, the word length and the requirement to perform articulatory suppression were manipulated. In both tasks, there were again significant word length effects and also significant reductions in recall when the participants were required to perform articulatory suppression. Significant word length effects were also obtained on both tasks when the list length was shortened to 6 items (Experiment 4) and lengthened to 12 items (Experiment 5).

We interpret these similar effects on recall by variables assumed to affect rehearsal as being consistent with accounts of memory that provide explanations of both tasks (e.g., Anderson et al., 1998; Brown et al., 2008; Brown et al., 2007), and we believe that our findings provide motivation for theories that currently explain only one of the two tasks to be extended to account for both free recall and ISR.

\section{Different Serial Position Curves: The Role of Rehearsal and Output Order}

The most marked differences between free recall and ISR were in the shapes of the serial position curves on the two tasks. Free recall was generally characterized by primacy and extended recency effects, whereas ISR was generally characterized by extended primacy effects. Our data therefore strongly suggest that very different shaped serial position curves can be obtained despite near identical patterns of rehearsal. It is necessary, therefore, to reflect upon the function of rehearsal in free recall and ISR.

Our supposition is that the shapes of the serial position curves are fundamentally determined by both the accessibility of individual list items and the output order of the words recalled at test. We assume that episodic memory is a continuum spanning from the most recently experienced events back to the least recently experienced events, and that presented items and their rehearsals can be considered separate ordered events on that continuum. We assume that the accessibility of a list item for recall increases as a function of the number, recency, and distribution of the rehearsals of that item (Tan \& Ward, 2000). During study (or test), an item that is accessible can be rehearsed (or recalled), and the rehearsal (or recall) of an item further increases its accessibility by creating another, more distributed, and more recently experienced memory event.

We assume that participants have some control (at least with list lengths of 6-12) over which words they choose to output first, and they may prefer to start recall with words toward the end of the list (when under free recall instructions) or may initiate recall with the first word in the list (as instructed in ISR and as sometimes selected in free recall).

However, participants are free to output at test only those words that are currently accessible. As additional 
words are added to the list (or are rehearsed or recalled), early list items that are not rehearsed will become less accessible (see, e.g., Tan \& Ward, 2008; Ward, 2002). The opportunity to rehearse early items is reduced if the words are studied at a fast presentation rate, if the words are long words, and/or if the words are encoded during articulatory suppression. The role of rehearsal is, therefore, to maintain the accessibility of (early) list items during study until the time of test, but which words are subsequently recalled is determined by both accessibility and output order.

In line with our supposition, we have demonstrated that participants tend to rehearse early list items most frequently, and that participants' ability to rehearse decreases with longer words and faster presentation rates and (so we assume) under articulatory suppression. In line with our proposal, we predict large reductions in primacy in ISR and free recall when the opportunity to rehearse is reduced. Such cases include (1) a reduction in primacy in ISR and free recall with faster presentation rates, as in Experiment 2; (2) a reduction in primacy in ISR and free recall with longer words, as in Experiments 2-5; and (3) a reduction in primacy in ISR and free recall with short words under articulatory suppression, as in Experiment 3. We additionally predict that there will be little or no reduction by these same factors on the recall of words that are rarely rehearsed, such as (1) the items presented toward the end of the list and (2) long words presented at a moderate rate under articulatory suppression. Furthermore, the accessibility of the first words in the list will be reduced with longer lists, relative to shorter lists, a finding consistent with both the free recall and ISR output orders.

Once recall has been initiated, we assume that participants tend to recall in a forward order. There is clear evidence from both the ISR data and free recall data that the recall of one item effectively cues recall of the immediately succeeding item (e.g., Howard \& Kahana, 1999; Nairne, Ceo, \& Reysen, 2007) but may impair recall of other list items (Nairne et al., 2007), a form of output interference (for other examples of output interference, see, e.g., Bhatarah et al., 2008; Cowan, Saults, \& Brown, 2004; Cowan, Saults, Elliott, \& Moreno, 2002; Dalezman, 1976; Oberauer, 2003; Tan \& Ward, 2007).

\section{Recall When Rehearsal Is Restricted}

According to our supposition, recall from the beginning of the list is still possible (although reduced) when the opportunity to rehearse is greatly reduced or eliminated (e.g., when the list is presented at a fast rate, or when it is presented under articulatory suppression). In these situations, it is assumed that the accessibilities at time of test will simply reflect the accessibilities of the originally encoded events.

However, in order to explain word length effects under articulatory suppression and at fast presentation rates, one also has to assume that some aspects of word length effects are not entirely due to differential rehearsal (cf. similar data reported by Baddeley \& Lewis, 1984; Coltheart \& Langdon, 1998). Our data could be considered to be consistent with the importance of the output delay at recall (e.g., Dosher \& Ma, 1998) and/or the contribution of word complexity to the word length effect (Neath \& Nairne, 1995). However, regardless of the preferred alternative, our data must surely be consistent with factors other than solely differential rehearsal's contributing to the word length effect (e.g., Brown \& Hulme, 1995; Hulme et al., 2006; Hulme et al., 2004; Lewandowsky \& Farrell, 2000; Lewandowsky \& Oberauer, 2008; Nairne, 2002; Neath \& Brown, 2006; Tehan \& Tolan, 2007).

\section{Implications for Theories of Free Recall and ISR}

Suppose that one is willing to entertain the notion that the rehearsal and recall processes used in ISR are the same as (or at least similar to) those used in free recall for short lists. How, then, would this impact on the respective ISR and free recall literatures?

First, one might consider extending recent accounts of free recall (e.g., Brown et al., 2007; Howard \& Kahana, 2002; Laming, 2006, 2008) to explain rehearsal and recall in ISR. All three recent accounts of free recall offer some appealing characteristics. The SIMPLE model of Brown et al. (2007) assumes that the processes underpinning free recall are the very same as those underpinning ISR and, hence, might be well placed to account for the similarities in the effects of the variables manipulated in these experiments on recall, even though it does not as yet have a process account of rehearsal or output order. The temporal context model of Howard and Kahana (2002) predicts that retrieval in free recall will tend to be forward ordered, even though participants are free to recall in any order that they wish. It may be that forward-ordered recall is a general property of recall at all time scales and tasks, and the recent data by Bhatarah et al. (2008) suggest that the degree of forward-ordered recall can be just as great in the free recall of short lists of words as in the ISR of similar lists. Laming's $(2006,2008)$ account of free recall assumes that the processes underpinning rehearsal are the same as the processes underpinning recall, and it may be that this hypothesis can be extended to both free recall and ISR, which might share common rehearsal and retrieval mechanisms, at least for short lists. All three of these accounts of free recall assume that there is no distinct STS and LTS, and this may prove to be an advantageous starting point, given the lack of trade-offs observed between concurrent free recall and ISR (Baddeley \& Hitch, 1974, 1977; Bhatarah et al., 2006).

An alternative approach is to examine whether theories of ISR, such as the phonological loop (Baddeley, 1986, 2007), could be extended to account for rehearsal and recall phenomena in free recall. For example, the phonological loop could be responsible for the forward order rehearsal of items in free recall and ISR; such rehearsal will serve to enhance the recency of the rehearsed items at the time that retrieval is required. The finding of similar effects of phonological variables on rehearsal in free recall in this study is clearly in line with a common phonological loop account of rehearsal in free recall and ISR.

However, one potential limitation in extending dualstore theories of free recall (e.g., Davelaar et al., 2005; Gillund \& Shiffrin, 1984; Raaijmakers \& Shiffrin, 1981) or STS accounts of ISR to both tasks is that it seems un- 
likely that both ISR and recency in free recall can be accommodated by a common STS (Baddeley \& Hitch, 1974, 1977; Bhatarah et al., 2006). This limitation can be circumvented if one assumes that recency and rehearsal are supported by different mechanisms.

\section{Situations in Which the Patterns of Rehearsal on the Two Tasks May Look Rather Different}

The data reported here are from lists of 6 to 12 unrelated words, and the claims made for similarities between rehearsal and recall on the two tasks might be unrealistic for longer lists or for semantically related lists. The list length is important in determining the output order and may also be important in determining the rehearsal strategy for items. It would seem likely, for example, that one would have expected rather different rehearsal strategies between free recall and ISR if we had used much longer lists or had used lists of semantically related items: Participants wishing to maximize the numbers of words recalled irrespective of output order (free recall) may well subjectively organize the list of words (e.g., by semantic category or by rehearsing different subsets of the list during study), whereas participants endeavoring to maintain the original list order might seek to maintain the input order of the early subset of items in the patterns of rehearsals.

\section{Summary and Conclusions}

In summary, this study has provided evidence that free recall and ISR share many similar aspects of the rehearsal and recall processes. Variables such as presentation rate that implicate a role for rehearsal in free recall were shown to have similar effects in ISR, and variables such as word length and articulatory suppression that implicate a role for rehearsal in ISR were shown to have similar effects in free recall. Direct observation of the rehearsals made using the overt rehearsal method provided further evidence for highly similar rehearsal processes in the two tasks.

Our preferred interpretation of these findings is that participants use the same retrieval mechanisms when rehearsing and recalling in ISR and free recall (at least for relatively short lists of unrelated items). We wish to highlight that participants possess a degree of control over the order of rehearsal and recall, so that grossly similar patterns of rehearsals can precede rather different output orders at recall. Although we are inexact in our specifications, we propose that the general retrieval mechanism(s) should possess the general properties of recency and forward-ordered recall and should be sensitive to output interference. One potential benefit of our approach is that it promotes cross-talk between the theories and findings of free recall and ISR memory tasks and seeks to redress the current divergence between the two literatures, which, after all, seek to explain rather similar immediate memory tasks.

\section{AUTHOR NOTE}

These experiments were conducted as part of the $\mathrm{PhD}$ thesis of the first author (supported by ESRC Research Studentship R42200134058) under the supervision of the second author. Correspondence concerning this article should be addressed to P. Bhatarah, Department of Psychology, London Metropolitan University, Calcutta House, 1 Old
Castle Street, London E1 7NT, England (e-mail: p.bhatarah@londonmet ac.uk).

\section{REFERENCES}

Anderson, J. R., Bothell, D., Lebiere, C., \& Matessa, M. (1998). An integrated theory of list memory. Journal of Memory \& Language, 38, 341-380.

AtKinson, R. C., \& Shiffrin, R. M. (1968). Human memory: A proposed system and its control processes. In K. W. Spence \& J. T. Spence (Eds.), The psychology of learning and motivation (Vol. 2, pp. 89195). New York: Academic Press.

AtKinson, R. C., \& Shiffrin, R. M. (1971). The control of short-term memory. Scientific American, 225, 82-90.

Avons, S. E., Wright, K. L., \& PAmmer, K. (1994). The word-length effect in probed and serial recall. Quarterly Journal of Experimental Psychology, 47A, 1047-1054.

BADDELEY, A. D. (1986). Working memory. Oxford: Oxford University Press, Clarendon Press.

BAdDELEy, A. [D.] (2000). The episodic buffer: A new component of working memory? Trends in Cognitive Sciences, 4, 417-423.

Baddeley, A. [D.] (2007). Working memory, thought, and action. Oxford: Oxford University Press.

Baddeley, A. D., \& Hitch, G. J. (1974). Working memory. In G. A. Bower (Ed.), The psychology of learning and motivation: Advances in research (Vol. 8, pp. 47-90). New York: Academic Press.

Baddeley, A. D., \& Hitch, G. J. (1977). Recency re-examined. In S. Dornic (Ed.), Attention and performance VI (pp. 647-667). Hillsdale, NJ: Erlbaum.

BADDEley, A. [D.], \& LARSEN, J. D. (2003). The disruption of STM: A response to our commentators. Quarterly Journal of Experimental Psychology, 56A, 1301-1306.

Baddeley, A. D., \& Larsen, J. D. (2007). The phonological loop unmasked? A comment on the evidence for a "perceptual-gestural" alternative. Quarterly Journal of Experimental Psychology, 60, 497-504.

BADDELEY, A. [D.], \& LEWIS, V. (1984). When does rapid presentation enhance digit span? Bulletin of the Psychonomic Society, 22, 403405 .

BadDEley, A. [D.], LeWIS, V., \& VAllar, G. (1984). Exploring the articulatory loop. Quarterly Journal of Experimental Psychology, 36A, 233-252.

Baddeley, A. D., Thomson, N., \& Buchanan, M. (1975). Word length and the structure of short-term memory. Journal of Verbal Learning \& Verbal Behavior, 14, 575-589.

Bhatarah, P., WARD, G., \& TAN, L. (2006). Examining the relationship between immediate serial recall and free recall: The effect of concurrent task performance. Journal of Experimental Psychology: Learning, Memory, \& Cognition, 32, 215-229.

Bhatarah, P., Ward, G., \& TAN, L. (2008). Examining the relationship between free recall and immediate serial recall: The serial nature of recall and the effect of test expectancy. Memory \& Cognition, 36, 20-34.

Bireta, T. J., Neath, I., \& Surprenant, A. M. (2006). The syllablebased word length effect and stimulus set specificity. Psychonomic Bulletin \& Review, 13, 434-438.

Brodie, D. A., \& Murdock, B. B. (1977). Effect of presentation time on nominal and functional serial-position curves of free recall. Journal of Verbal Learning \& Verbal Behavior, 16, 185-200.

Brown, G. D. A., Chater, N., \& Neath, I. (2008). Serial and free recall: Common effects and common mechanisms? A reply to Murdock (2008). Psychological Review, 115, 781-785.

Brown, G. D. A., \& Hulme, C. (1995). Modeling item length effects in memory span: No rehearsal needed? Journal of Memory \& Language, 34, 594-621.

Brown, G. D. A., Neath, I., \& Chater, N. (2007). A temporal ratio model of memory. Psychological Review, 114, 539-576.

Brown, G. D. A., Preece, T., \& Hulme, C. (2000). Oscillator-based memory for serial order. Psychological Review, 107, 127-181.

Burgess, N., \& Hitch, G. (1992). Toward a network model of the articulatory loop. Journal of Memory \& Language, 31, 429-460.

Burgess, N., \& Hitch, G. (1999). Memory for serial order: A network model of the phonological loop and its timing. Psychological Review, 106, 551-581. 
Campoy, G., \& Baddeley, A. (2008). Phonological and semantic strategies in immediate serial recall. Memory, 16, 329-340.

Caplan, D., Rochon, E., \& Waters, G. (1992). Articulatory and phonological determinants of word length effects in span tasks. Quarterly Journal of Experimental Psychology, 45A, 177-192.

Coltheart, V., \& Langdon, R. (1998). Recall of short word lists presented visually at fast rates: Effects of phonological similarity and word length. Memory \& Cognition, 26, 330-342.

Conrad, R., \& Hille, B. A. (1958). The decay theory of immediate memory and paced recall. Canadian Journal of Psychology, 12, 1-6.

Cowan, N. (2005). Attention and memory: An integrated framework. New York: Oxford University Press.

Cowan, N., Saults, J. S., \& Brown, G. D. A. (2004). On the auditory modality superiority effect in serial recall: Separating input and output factors. Journal of Experimental Psychology: Learning, Memory, \& Cognition, 30, 639-644.

Cowan, N., Saults, J. S., Elliott, E. M., \& Moreno, M. V. (2002). Deconfounding serial recall. Journal of Memory \& Language, 46, 153-177.

Dalezman, J. J. (1976). Effects of output order on immediate, delayed, and final free recall performance. Journal of Experimental Psychology: Human Learning \& Memory, 2, 597-608.

Davelaar, E. J., Goshen-Gottstein, Y., Ashkenazi, A., HaArmann, H. J., \& UsHer, M. (2005). The demise of short-term memory revisited: Empirical and computational investigations of recency effects. Psychological Review, 112, 3-42.

Dosher, B. A., \& MA, J. J. (1998). Output loss or rehearsal loop? Output-time versus pronunciation-time limits in immediate recall for forgetting-matched materials. Journal of Experimental Psychology: Learning, Memory, \& Cognition, 24, 316-325.

FARrell, S., \& LeWANDowsky, S. (2002). An endogenous distributed model of ordering in serial recall. Psychonomic Bulletin \& Review, 9, 59-79.

Fischler, I., Rundus, D., \& AtKInson, R. C. (1970). Effects of overt rehearsal procedures on free recall. Psychonomic Science, 19, 249-250.

Friendly, M., Franklin, P. E., Hoffman, D., \& Rubin, D. C. (1982). The Toronto Word Pool: Norms for imagery, concreteness, orthographic variables, and grammatical usage for 1,080 words. Behavior Research Methods \& Instrumentation, 14, 375-399.

Gillund, G., \& Shiffrin, R. M. (1984). A retrieval model for both recognition and recall. Psychological Review, 91, 1-67.

Glanzer, M. (1972). Storage mechanisms in recall. In G. H. Bower (Ed.), The psychology of learning and motivation: Advances in research and theory (Vol. V, pp. 129-193). New York: Academic Press.

Glanzer, M., \& Cunitz, A. R. (1966). Two storage mechanisms in free recall. Journal of Verbal Learning \& Verbal Behavior, 5, 351-360.

Golomb, J. D., Peelle, J. E., AdDis, K. M., Kahana, M. J., \& WingFIELD, A. (2008). Effects of adult aging on utilization of temporal and semantic associations during free and serial recall. Memory \& Cognition, 36, 947-956.

Henson, R. N. A. (1998). Short-term memory for serial order: The startend model of serial recall. Cognitive Psychology, 36, 73-137.

Howard, M. W., \& Kahana, M. J. (1999). Contextual variability and serial position effects in free recall. Journal of Experimental Psychology: Learning, Memory, \& Cognition, 25, 1-19.

Howard, M. W., \& KaHANA, M. J. (2002). A distributed representation of temporal context. Journal of Mathematical Psychology, 46, 269-299.

Hulme, C., Neath, I., Stuart, G., Shostak, L., Suprenant, A. M., \& Brown, G. D. A. (2006). The distinctiveness of the word-length effect. Journal of Experimental Psychology: Learning, Memory, \& Cognition, 32, 586-594.

Hulme, C., Suprenant, A. M., Bireta, T. J., Stuart, G., \& Neath, I. (2004). Abolishing the word length effect. Journal of Experimental Psychology: Learning, Memory, \& Cognition, 30, 98-106.

KuČERA, H., \& FranCIS, W. N. (1967). Computational analysis of presentday American English. Providence, RI: Brown University Press.

LAmING, D. (2006). Predicting free recalls. Journal of Experimental Psychology: Learning, Memory, \& Cognition, 32, 1146-1163.

LAMING, D. (2008). An improved algorithm for predicting free recalls. Cognitive Psychology, 57, 179-219.
La Pointe, L. B., \& Engle, R. W. (1990). Simple and complex word spans as measures of working memory capacity. Journal of Experimental Psychology: Learning, Memory, \& Cognition, 16, 1118-1133.

Larsen, J. D., \& Baddeley, A. D. (2003). Disruption of verbal STM by irrelevant speech, articulatory suppression, and manual tapping: Do they have a common source? Quarterly Journal of Experimental Psychology, 56A, 1249-1268.

LEVY, B. A. (1971). The role of articulation in auditory and visual shortterm memory. Journal of Verbal Learning \& Verbal Behavior, 10, 123-132.

LEWANDOWSKY, S., \& FARRELL, S. (2000). A redintegration account of the effects of speech rate, lexicality, and word frequency in immediate serial recall. Psychological Research, 63, 163-173.

LewANDOWsky, S., \& OBerauer, K. (2008). The word-length effect provides no evidence for decay in short-term memory. Psychonomic Bulletin \& Review, 15, 875-888.

Longoni, A. M., Richardson, J. T. E., \& Aiello, A. (1993). Articulatory rehearsal and phonological storage in working memory. Memory \& Cognition, 21, 11-22.

Lovatt, P., Avons, S. E., \& Masterson, J. (2000). The word-length effect and disyllabic words. Quarterly Journal of Experimental Psychology, 53A, 1-22.

Lovatt, P., Avons, S. E., \& Masterson, J. (2002). Output decay in immediate serial recall: Speech time revisited. Journal of Memory \& Language, 46, 227-243.

Mackworth, J. F. (1962). Presentation rate and immediate memory. Canadian Journal of Psychology, 16, 42-47.

Miller, G. A. (1956). The magic number seven, plus or minus two: Some limits on our capacity for processing information. Psychological Review, 63, 81-93.

Modigliani, V., \& Hedges, D. G. (1987). Distributed rehearsals and the primacy effect in single-trial free recall. Journal of Experimental Psychology: Learning, Memory, \& Cognition, 13, 426-436.

Mueller, S. T., Seymour, T. L., Kieras, D. E., \& Meyer, D. E. (2003). Theoretical implications of articulatory duration, phonological similarity, and phonological complexity in verbal working memory. Journal of Experimental Psychology: Learning, Memory, \& Cognition, 29, 1353-1380.

Murdock, B. B., JR. (1962). The serial position effect of free recall. Journal of Experimental Psychology, 64, 482-488.

Murdock, B. [B., JR.] (2008). Issues with the SIMPLE model: Comment on Brown, Neath, and Chater (2007). Psychological Review, 115, 779-780.

MurRAY, D. J. (1968). Articulation and acoustic confusability in shortterm memory. Journal of Experimental Psychology, 78, 679-684.

NAIRNE, J. S. (1988). A framework for interpreting recency effects in immediate serial recall. Memory \& Cognition, 16, 343-352.

NAIRNE, J. S. (2002). Remembering over the short-term: The case against the standard model. Annual Review of Psychology, 53, 53-81.

Nairne, J. S., Ceo, D. A., \& Reysen, M. B. (2007). The mnemonic effects of recall on immediate retention. Memory \& Cognition, 35, 191-199.

Neath, I., \& Brown, G. D. A. (2006). SIMPLE: Further applications of a local distinctiveness model of memory. In B. H. Ross (Ed.), The psychology of learning and motivation (Vol. 46, pp. 201-243). San Diego: Academic Press.

NeATH, I., \& NaIRne, J. S. (1995). Word-length effects in immediate memory: Overwriting trace decay theory. Psychonomic Bulletin \& Review, 2, 429-441

OBERAUER, K. (2003). Understanding serial position curves in shortterm recognition and recall. Journal of Memory \& Language, 49, 469483.

PAGE, M. P. A., \& Norris, D. (1998). The primacy model: A new model of immediate serial recall. Psychological Review, 105, 761-781.

Posner, M. I. (1964). Rate of presentation and order of recall in immediate memory. British Journal of Psychology, 55, 303-306.

Quinlan, P. T. (1992). The Oxford psycholinguistic database. Oxford: Oxford University Press, Oxford Electronic Publishing.

RAAIJMAKERS, J. G. W., \& SHIFFrin, R. M. (1981). Search of associative memory. Psychological Review, 88, 93-134.

Richardson, J. T. E., \& BadDELEy, A. D. (1975). The effect of articula- 
tory suppression in free recall. Journal of Verbal Learning \& Verbal Behavior, 14, 623-629.

Rundus, D. (1971). Analysis of rehearsal processes in free recall. Journal of Experimental Psychology, 89, 63-77.

Rundus, D., \& AtKinson, R. C. (1970). Rehearsal processes in free recall: A procedure for direct observation. Journal of Verbal Learning \& Verbal Behavior, 9, 99-105

Russo, R., \& Grammatopoulou, N. (2003). Word length and articulatory suppression affect short-term and long-term recall tasks. Memory \& Cognition, 31, 728-737.

Salthouse, T. A. (1980). Age and memory: Strategies for localizing the loss. In L. W. Poon, J. L. Fozard, L. S. Cermack, D. Arenberg, \& L. W. Thompson (Eds.), New directions in memory and aging: Proceedings of the George A. Talland Memorial Conference (pp. 47-65). Hillsdale, NJ: Erlbaum.

SCHWEIKERT, R., \& BorUfF, B. (1986). Short-term memory capacity: Magic number or magic spell? Journal of Experimental Psychology: Learning, Memory, \& Cognition, 12, 419-425.

SERVICE, E. (1998). The effect of word length on immediate serial recall depends on phonological complexity, not articulatory duration. Quarterly Journal of Experimental Psychology, 51A, 283-304.

TAN, L., \& WARD, G. (2000). A recency-based account of primacy effects in free recall. Journal of Experimental Psychology: Learning, Memory, \& Cognition, 26, 1589-1625.

TAN, L., \& WARD, G. (2007). Output order in immediate serial recall. Memory \& Cognition, 35, 1093-1106.
TAN, L., \& WARD, G. (2008). Rehearsal in immediate serial recall. Psychonomic Bulletin \& Review, 15, 535-542.

Tehan, G., \& Tolan, G. A. (2007). Word length effects in long-term memory. Journal of Memory \& Language, 56, 35-48.

Tolan, G. A., \& TeHan, G. (2005). Is spoken duration a sufficient explanation of the word length effect? Memory, 13, 372-379.

WARD, G. (2001). A critique of the working memory model. In J. Andrade (Ed.), Working memory in perspective (pp. 219-239). Hove, U.K.: Psychology Press.

WARD, G. (2002). A recency-based account of the list length effect in free recall. Memory \& Cognition, 30, 885-892.

Ward, G., TAN, L., \& Bhatarah, P. (2008). The roles of short-term verbal memory in free and serial recall: Towards a recency-based perspective. In A. S. C. Thorn \& M. P. A. Page (Eds.), Interactions between short-term and long-term memory in the verbal domain (pp. 4462). Hove, U.K.: Psychology Press.

WARD, G., TAN, L., \& GRENFell-Essam, R. (2009, July). List length effects and output order in free recall: Implications for the relationship between immediate free and serial recall. Paper presented at the meeting of the Experimental Psychology Society, York, U.K.

WATKINS, M. J. (1972). Locus of the modality effect in free recall. Journal of Verbal Learning \& Verbal Behaviour, 11, 644-648.

(Manuscript received May 12, 2008;

revision accepted for publication February 6, 2009.) 